\title{
Development, Scope, and Mechanism of the Palladium-Catalyzed Intramolecular Hydroalkylation of 3-Butenyl $\beta$-Diketones
}

\author{
Hua Qian, Tao Pei, and Ross A. Widenhoefer* \\ P. M. Gross Chemical Laboratory \\ Duke University
}

Durham, NC 27708-0346

\section{Supporting Information}

Experimental procedures, analytical and spectroscopic data for new compounds and cyclohexanones (22 pages). 


\section{Experimental}

General Methods. All catalytic reactions were performed under an atmosphere of dry nitrogen at room temperature. NMR spectra were obtained on a Varian spectrometer operating at $400 \mathrm{MHz}$ for ${ }^{1} \mathrm{H}$ $\mathrm{NMR}$ and $100 \mathrm{MHz}$ for ${ }^{13} \mathrm{C} \mathrm{NMR}$ in $\mathrm{CDCl}_{3}$ unless otherwise noted. Alkenyl $\beta$-diketones exist in solution as mixtures of enol and keto tautomers; only resonances corresponding to the predominant enol tautomer are given unless specified otherwise. For deuterated isotopomers, only resonances that differ from the corresponding unlabeled isotopomer are reported; isotopic shifts (IS) are reported relative to the appropriate resonance in the corresponding unlabeled isotopomer. IR spectra were obtained on a Bomen MB-100 FT IR spectrometer. Gas chromatography was performed on a HP 5890 gas chromatography equipped with a $25 \mathrm{~m}$ polydimethylsiloxane capillary column. Flash column chromatography was performed employing 200-400 mesh silica gel (EM). Thin layer chromatography (TLC) was performed on silica gel $60 \mathrm{~F}_{254}$ eluting with a 5:1 mixture of hexanes and ethyl acetate unless noted otherwise. Elemental analyses were performed by Complete Analysis Laboratories (Parsippany, NJ). 1,4-Dioxane, acetonitrile, and DMSO (Aldrich, anhydrous) were used as received, THF and ether were distilled from sodium benzophenone ketyl under nitrogen, methylene chloride was distilled from $\mathrm{CaH}_{2}$ under nitrogen.

$\mathrm{PdCl}_{2}(\mathrm{MeCN})_{2}(4)$ was purchased from Strem and used as received or was prepared from $\mathrm{PdCl}_{2}$ (Strem) employing a literature procedure. [1] Authentic samples of 5 (Aldrich), 13 (Lancaster), and 15 (Aldrich) were obtained from commercial suppliers. An authentic sample of $\mathbf{1 4}$ was synthesized employing the procedure of Reich.[2] Substrates 3,[3] 8-methyl-7-nonene-2,4-dione (Table 2, entry 11), [4] ethyl 3-oxo6-heptenoate (Table 2, entry 12),[5] methyl 3-oxo-6-heptenoate (Table 2, entry 13)[6] and all new substrates were prepared from the appropriate 1,3-diketone or $\beta$-keto ester and allylic halide or tosylate employing a modified published procedure unless otherwise noted. ${ }^{[7]} 3$-Methyl-7-octene-2,4-dione (Table 2, entry 5) ${ }^{[8]}$ and (E)-2,3-dibromo-1-phenylsulfonylpropene[9] were prepared according to the published procedures. 3,3-Dideuterioallyl methanesulfonate (S1) was prepared from 3,3-dideuterio-2-propene-1-ol[10] employing a procedure analogous to that used to synthesize unlabeled allyl methanesulfonate. ${ }^{[11]}(Z)-3$-Bromo-1,2dideuteriopropene (S2) and 3-bromo-3,3-dideuterio-1-propene (S3) were synthesized from reaction of $(Z)$ 2,3-dideuterio-2-propene-1-ol[12] and 1,1-dideuterio-2-propene-1-ol,[13] respectively, with 
dibromotriphenylphosphorane. 1-Deuterio-2-propene-1-ol was synthesized according to a literature procedure.[14]

\section{Synthesis of Alkenyl $\beta$-Diketones}

8-Nonene-3,5-dione (Table 2, entry 3). 8-Nonene-3,5-dione was synthesized from 5-hexen-2one and methyl propionate in $12 \%$ yield employing a modified literature procedure.[15] Enol:dione $=4: 1$. TLC: $\mathrm{R}_{f}=0.53 .{ }^{1} \mathrm{H}$ NMR: $\delta$ 5.96-5.85 (m, $\left.1 \mathrm{H}\right), 5.59(\mathrm{~s}, 1 \mathrm{H}), 5.18-5.07(\mathrm{~m}, 2 \mathrm{H}), 2.47(\mathrm{~m}, 4 \mathrm{H}), 2.41$ $(\mathrm{q}, J=7.6 \mathrm{~Hz}, 2 \mathrm{H}), 1.23(\mathrm{t}, J=7.6 \mathrm{~Hz}, 3 \mathrm{H}) .{ }^{13} \mathrm{C}\left\{{ }^{1} \mathrm{H}\right\} \mathrm{NMR}: \delta 195.6,193.6,137.2,115.8,98.8,37.9$, 31.8, 29.8, 10.0. IR (neat, $\mathrm{cm}^{-1}$ ): 3078, 2977, 2938, 2918, 1726, 1613, 1444, 1416, 1377, 1352, 1323, 1202, 1142, 1064, 994, 914. Anal. calcd (found) for $\mathrm{C}_{9} \mathrm{H}_{14} \mathrm{O}_{2}$ : C, 70.10 (70.21); H, 9.15 (9.25).

2,2-Dimethyl-8-nonene-3,5-dione (Table 2, entry 4). Enol:dione $=13: 1$. TLC: $\mathrm{R}_{f}=0.71 .{ }^{1} \mathrm{H}$ NMR: $\delta$ 5.87-5.77 (m, $1 \mathrm{H}), 5.60(\mathrm{~s}, 1 \mathrm{H}), 5.08-4.97(\mathrm{~m}, 2 \mathrm{H}), 2.43-2.35(\mathrm{~m}, 4 \mathrm{H}), 1.16(\mathrm{~s}, 9 \mathrm{H})$. ${ }^{13} \mathrm{C}\left\{{ }^{1} \mathrm{H}\right\} \mathrm{NMR}: \delta$ 200.4, 195.1, 137.3, 115.7, 95.5, 51.8, 38.4, 29.9, 27.6. IR (neat, $\mathrm{cm}^{-1}$ ): 3079, 2968, 2927, 2874, 1697, 1620, 1455, 1362, 1275, 1221, 1133, 1087. Anal. calcd (found) for $\mathrm{C}_{11} \mathrm{H}_{18} \mathrm{O}_{2}$ : $\mathrm{C}$, 72.49 (72.35); H, 9.95 (9.91).

3-Benzyl-7-octene-2,4-dione (Table 2, entry 6). 3-Benzyl-7-octene-2,4-dione was synthesized

from 7-octene-2,4-dione (3) and benzyl bromide in 35\% yield employing a modified literature procedure.[16] Enol:dione $=1: 1.3$. TLC: $\mathrm{R}_{f}=0.43 .{ }^{1} \mathrm{H}$ NMR: $\delta$ 7.30-7.14 (m, $\left.5 \mathrm{H}\right), 5.69(\mathrm{tdd}, J=6.4$, 10.4, 17.2 Hz, $1 \mathrm{H}), 4.96-4.92(\mathrm{~m}, 2 \mathrm{H}), 4.00$ (t, $J=7.6 \mathrm{~Hz}, 1 \mathrm{H}), 3.15$ (dd, J = 4.0, 7.6 Hz, $2 \mathrm{H}), 2.46-2.36$ $(\mathrm{m}, 2 \mathrm{H}), 2.27-2.22(\mathrm{~m}, 2 \mathrm{H}), 2.12(\mathrm{~s}, 3 \mathrm{H}) .{ }^{13} \mathrm{C}\left\{{ }^{1} \mathrm{H}\right\} \mathrm{NMR}: \delta 205.2,203.8,136.8,129.1,129.0,127.8$ 127.1, 115.9, 69.8, 42.4. 34.7, 32.7, 27.5. IR (neat, $\mathrm{cm}^{-1}$ ): 3083, 3072, 3028, 2933, 1728, 1697, 1643, $1603,1495,1454,1358,1259,1183,1162,1092,995,915$. Anal. calcd (found) for $\mathrm{C}_{15} \mathrm{H}_{18} \mathrm{O}_{2}$ : C, 78.23 (78.07); H, 7.88 (8.02).

cis-7-Nonene-2,4-dione (Table 2, entry 7). A suspension of Lindlar's catalyst (115 mg, $5 \mathrm{~mol}$ $\%)$ and 7-nonyne-2,4-dione $(780 \mathrm{mg}, 5.13 \mathrm{mmol})$ in acetone $(20 \mathrm{~mL})$ was stirred under hydrogen $(1 \mathrm{~atm})$ overnight at room temperature, concentrated, and chromatographed (hexanes-EtOAc $=125: 1$ ) to give cis-7nonene-2,4-dione (540 mg, 69\%) as a pale yellow oil. 
For 7-nonyne-2,4-dione: Enol:dione $=5: 1$. TLC: $\mathrm{R}_{f}=0.35 .{ }^{1} \mathrm{H}$ NMR $: \delta 5.52(\mathrm{~s}, 1 \mathrm{H}), 2.48$ $2.42(\mathrm{~m}, 4 \mathrm{H}), 2.05$ (s, $3 \mathrm{H}), 1.76-1.73(\mathrm{~m}, 3 \mathrm{H}) .{ }^{13} \mathrm{C}\left\{{ }^{1} \mathrm{H}\right\}$ NMR: $\delta$ 193.1, 190.8, 100.2, 77.7, 76.8, 38.1, 25.0, 15.2, 3.8. IR (neat, $\mathrm{cm}^{-1}$ ): 2969, 2919, 1857, 2357, 1729, 1713, 1620, 1417, 1360, 1236, 1132, 1005. Anal. calcd (found) for $\mathrm{C}_{9} \mathrm{H}_{12} \mathrm{O}_{2}:$ C, 71.03 (71.04); H, 7.95 (7.90).

For cis-7-nonene-2,4-dione. Enol:dione $=5: 1 . \quad \mathrm{TLC}: \mathrm{R}_{f}=0.46 .{ }^{1} \mathrm{H}$ NMR: $\delta$ 5.53-5.46 (m, 1 $\mathrm{H}), 5.50(\mathrm{~s}, 1 \mathrm{H}), 5.38-5.32(\mathrm{~m}, 1 \mathrm{H}), 2.38-2.30(\mathrm{~m}, 4 \mathrm{H}), 2.05(\mathrm{~s}, 3 \mathrm{H}), 1.63-1.60(\mathrm{~m}, 3 \mathrm{H}) .{ }^{13} \mathrm{C}\left\{{ }^{1} \mathrm{H}\right\}$ NMR: $\delta$ 193.9, 191.7, 128.8, 125.7, 100.2, 38.3, 25.3, 23.3, 13.1. IR (neat, $\mathrm{cm}^{-1}$ ): 3015, 2958, 2920, $2860,1709,1613,1443,1359,1234,1138,1096,1030,998,963,929$. Anal. calcd (found) for $\mathrm{C}_{9} \mathrm{H}_{14} \mathrm{O}_{2}$ : C, 70.10 (70.12); H, 9.15 (9.14).

trans-7-Nonene-2,4-dione (Table 2, entry 8). Enol:dione $=6: 1$. TLC: $\mathrm{R}_{f}=0.44 .{ }^{1} \mathrm{H}$ NMR: $\delta$ $5.48(\mathrm{~s}, 1 \mathrm{H}), 5.47-5.36(\mathrm{~m}, 2 \mathrm{H}), 2.34-2.22(\mathrm{~m}, 4 \mathrm{H}), 2.04(\mathrm{~s}, 3 \mathrm{H}), 1.64-1.62(\mathrm{~m}, 3 \mathrm{H}) .{ }^{13} \mathrm{C}\left\{{ }^{1} \mathrm{H}\right\} \mathrm{NMR}$ : $\delta$ 194.0, 191.5, 129.6, 126.4, 100.2, 38.6, 28.8, 25.2, 18.2. IR (neat, $\mathrm{cm}^{-1}$ ): 3013, 2965, 2919, 2863, 1713, 1620, 1454, 1360, 1236, 1142, 967. HRMS (EI) calcd (found) for $\mathrm{C}_{9} \mathrm{H}_{14} \mathrm{O}_{2}\left(\mathrm{M}^{+}\right)$: 154.0994 (154.0995).

7-Dodecene-2,4-dione (Table 2, entry 9). Enol:dione = 5:1. ${ }^{1} \mathrm{H}$ NMR $(300 \mathrm{MHz}): \delta 5.47(\mathrm{~s}$, $1 \mathrm{H}), 5.45-5.35$ (m, $2 \mathrm{H}), 2.34-2.21(\mathrm{~m}, 4 \mathrm{H}), 2.03$ (s, $3 \mathrm{H}), 1.98-1.92$ (m, $2 \mathrm{H}), 1.31-1.24(\mathrm{~m}, 4 \mathrm{H}), 0.86(\mathrm{t}$, $J=7.4 \mathrm{~Hz}, 3 \mathrm{H}) .{ }^{13} \mathrm{C}\left\{{ }^{1} \mathrm{H}\right\} \mathrm{NMR}: \delta 193.8,191.5,132,0,128.2,100.1,38.4,32.3,31.8,28.7,25.1,22.3$, 14.1. IR (neat, $\mathrm{cm}^{-1}$ ): 3056, 2956, 2925, 2855, 1706, 1616, 1436, 1361, 1236, 1148, 967. HRMS (EI) calcd (found) for $\mathrm{C}_{12} \mathrm{H}_{20} \mathrm{O}_{2}\left(\mathrm{M}^{+}\right)$: 196.1463 (196.1460).

8-Phenyl-7-octene-2,4-dione (Table 2, entry 10). Enol:dione = 5:1. TLC: $\mathrm{R}_{f}=0.38 .{ }^{1} \mathrm{H}$ NMR: $\delta$ 7.31-7.23 (m, 4 H), 7.19-7.16 (m, 1 H), $6.39(\mathrm{~d}, J=16.0 \mathrm{~Hz}, 1 \mathrm{H}), 6.17(\mathrm{td}, J=6.8,16.0 \mathrm{~Hz}, 1$ H), 5.49 (s, $1 \mathrm{H}), 2.52-2.47(\mathrm{~m}, 2 \mathrm{H}), 2.46-2.40(\mathrm{~m}, 2 \mathrm{H}), 2.03(\mathrm{~s}, 3 \mathrm{H}) .{ }^{13} \mathrm{C}\left\{{ }^{1} \mathrm{H}\right\}$ NMR: $\delta$ 193.7, 191.4, 137.7, 131.2, 128.9, 128.8, 127.5, 126.4, 100.3, 38.3, 29.1, 25.2. IR (neat, $\mathrm{cm}^{-1}$ ): 3080, 3057, 3024, 2919, 2849, 1948, 1881, 1806, 1725, 1705, 1620, 1494, 1445, 1416, 1359, 1304, 1254, 1234, 1136, 1077, 965, 784, 744, 693. Anal. calcd (found) for $\mathrm{C}_{14} \mathrm{H}_{16} \mathrm{O}_{2}$ : C, 77.75 (77.81); $\mathrm{H}, 7.46$ (7.42).

Benzyl 3-oxo-6-heptenoate (Table 2, entry 14). Enol:dione $=1: 13 . \quad$ TLC: $\mathrm{R}_{f}=0.39 .{ }^{1} \mathrm{H}$ NMR : $\delta$ 7.37-7.35 (m, 5 H), 5.77 (tdd, $J=6.6,10.2,17.0 \mathrm{~Hz}, 1 \mathrm{H}), 5.18(\mathrm{~s}, 2 \mathrm{H}), 5.05-4.96(\mathrm{~m}, 2 \mathrm{H})$, $3.49(\mathrm{~s}, 2 \mathrm{H}), 2.62(\mathrm{t}, J=7.2 \mathrm{~Hz}, 2 \mathrm{H}), 2.36-2.30(\mathrm{~m}, 2 \mathrm{H}) .{ }^{13} \mathrm{C}\left\{{ }^{1} \mathrm{H}\right\} \mathrm{NMR}: \delta 201.9,167.2,136.7,135.5$, 
128.8, 128.7, 128.6, 115.8, 67.3, 49.5, 42.3, 27.6. IR (neat, $\mathrm{cm}^{-1}$ ): 3067, 3032, 2976, 2930, 1742, 1716, $1641,1497,1454,1409,1374,1313,1268,1223,1152,1081,997,915$. Anal. calcd (found) for $\mathrm{C}_{14} \mathrm{H}_{16} \mathrm{O}_{3}: \mathrm{C}, 72.39$ (72.16); H, 6.94 (6.78).

Synthesis of $(\boldsymbol{E})$-6-octene-2,4-dione [(E)-17]. (E)-17 was synthesized in 19\% overall yield from condensation of $(E)$-3-pentenal with acetone followed by Swern oxidation following a literature procedure (Scheme S1).[17]

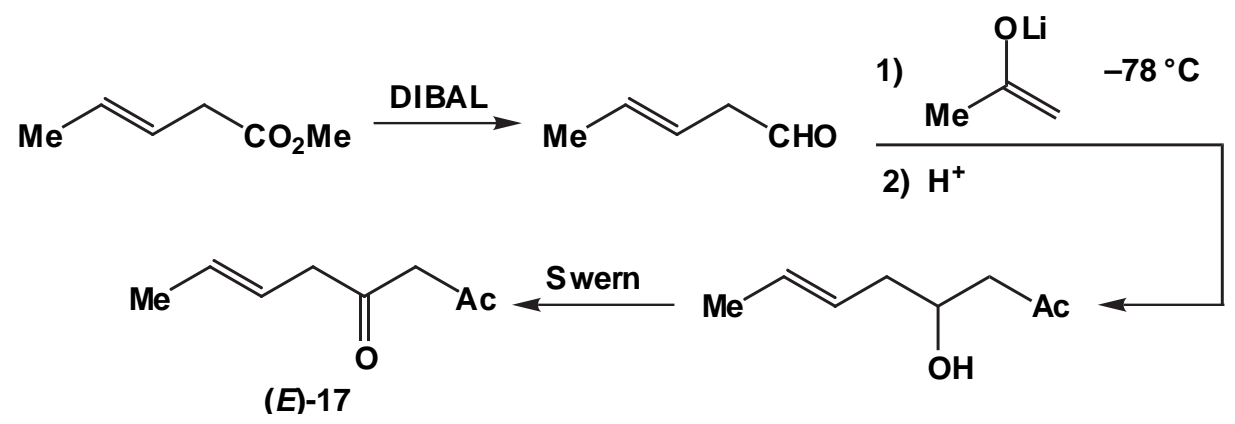

\section{Scheme S1}

(E)-3-Pentenal.[18] DIBAL $(10.8 \mathrm{~mL}, 60 \mathrm{mmol})$ was added dropwise to a solution of methyl $(E)$ 3-pentenoate ( $3.68 \mathrm{~mL}, 30 \mathrm{mmol})$ in $\mathrm{CH}_{2} \mathrm{Cl}_{2}(120 \mathrm{~mL})$ at $-78^{\circ} \mathrm{C}$ and stirred for $2 \mathrm{~h}$. The resulting mixture was treated sequentially with methanol $(10 \mathrm{~mL})$ and water $(20 \mathrm{~mL})$ and the resulting mixture was warmed to room temperature and poured into $1 \mathrm{~N}$ aqueous $\mathrm{HCl}(150 \mathrm{~mL})$. The layers were separated and the aqueous layer was extracted with $\mathrm{CH}_{2} \mathrm{Cl}_{2}(100 \mathrm{~mL})$. The combined organic extracts were dried $\left(\mathrm{MgSO}_{4}\right)$, filtered through a pad of silica gel, and concentrated to give a pale yellow oil $(1.70 \mathrm{~g}, 67 \%) .{ }^{1} \mathrm{H}$ NMR: $\delta$ $9.60(\mathrm{t}, J=2.0 \mathrm{~Hz}, 1 \mathrm{H}), 5.63-5.53(\mathrm{~m}, 1 \mathrm{H}), 5.51-5.43(\mathrm{~m}, 1 \mathrm{H}), 3.07-3.04(\mathrm{~m}, 2 \mathrm{H}), 1.69-1.66(\mathrm{qd}, J=1.2$, $6.4 \mathrm{~Hz}, 3 \mathrm{H}) .{ }^{13} \mathrm{C}\left\{{ }^{1} \mathrm{H}\right\}$ NMR: $\delta$ 200.6, 131.5, 120.6, 47.5, 18.3.

For $(\boldsymbol{E})$-4-hydroxy-6-octene-2-one: $28 \% . \quad \mathrm{TLC}: \mathrm{R}_{f}=0.12 .{ }^{1} \mathrm{H} \mathrm{NMR}: \delta 5.58-5.49(\operatorname{tqd}, J=$ $1.2,6.2,15.2 \mathrm{~Hz}, 1 \mathrm{H}), 5.45-5.37$ (qtd, $J=3.2,7.0,15.2 \mathrm{~Hz}, 1 \mathrm{H}), 4.08-4.01(\mathrm{~m}, 1 \mathrm{H}), 2.89$ (d, $J=3.2 \mathrm{~Hz}$, $1 \mathrm{H}), 2.64-2.49$ (m, $2 \mathrm{H}), 2.22-2.10$ (m, $2 \mathrm{H}), 2.17$ (s, $3 \mathrm{H}), 1.68-1.66$ (tdd, $J=1.0,2.4,6.4 \mathrm{~Hz}, 3 \mathrm{H}$ ). ${ }^{13} \mathrm{C}\left\{{ }^{1} \mathrm{H}\right\}$ NMR: $\delta$ 210.0, 129.2, 126.8, 67.6, 49.6, 40.0, 31.1, 18.4. IR (neat, $\mathrm{cm}^{-1}$ ): 3426, 1711, 1681. HRMS (EI): Calcd (found) for $\mathrm{C}_{8} \mathrm{H}_{12} \mathrm{O}\left(\mathrm{M}^{+}-\mathrm{H}_{2} \mathrm{O}\right)$ : 124.0888 (124.0884). 
For $(\boldsymbol{E})-17: 44 \%$. Enol: ketone $=6: 1$. TLC: $\mathrm{R}_{f}=0.32 .{ }^{1} \mathrm{H}$ NMR: $\delta 15.4($ br s, $1 \mathrm{H}), 5.62-5.44$ $(\mathrm{m}, 2 \mathrm{H}), 5.48(\mathrm{~s}, 1 \mathrm{H}), 2.96(\mathrm{~d}, J=6.8 \mathrm{~Hz}, 2 \mathrm{H}), 2.03(\mathrm{~s}, 3 \mathrm{H}), 1.69(\mathrm{dd}, J=1.4,6.2 \mathrm{~Hz}, 3 \mathrm{H}) .{ }^{13} \mathrm{C}\left\{{ }^{1} \mathrm{H}\right\}$ NMR: $\delta$ 193.6, 191.1, 130.1, 123.9, 99.7, 42.3, 25.0, 18.3. IR (neat, $\mathrm{cm}^{-1}$ ): 3405, 3028, 1726, 1710, 1613. Anal. Calcd (found) for $\mathrm{C}_{8} \mathrm{H}_{12} \mathrm{O}_{2}$ : C, 68.54 (68.35); $\mathrm{H}, 8.63$ (8.41).

Synthesis of $(\boldsymbol{E})-5$-octene-2,4-dione $[(\boldsymbol{E})-18]$. $(E)$-18 was synthesized starting from $(E)-2$ pentenal employing a two-step procedure similar to that used to synthesize $(E)-17$.

(E)-4-Hydroxy-5-octene-2-one. $76 \%$. TLC (hexanes-ether $=1: 1$ ): $\mathrm{R}_{f}=0.62 .{ }^{1} \mathrm{H}$ NMR: $\delta$ $5.69(\mathrm{dtd}, J=1.2,6.4,15.2 \mathrm{~Hz}, 1 \mathrm{H}), 5.41(\mathrm{tdd}, J=1.6,6.6,15.4 \mathrm{~Hz}, 1 \mathrm{H}), 4.50-4.44(\mathrm{~m}, 1 \mathrm{H}), 3.04-3.01$ $(\mathrm{m}, 1 \mathrm{H}), 2.61(\mathrm{~d}, J=6.4 \mathrm{~Hz}, 1 \mathrm{H}), 2.60(\mathrm{~s}, 1 \mathrm{H}), 2.14(\mathrm{~s}, 3 \mathrm{H}), 2.03-1.96(\mathrm{~m}, 2 \mathrm{H}), 0.94(\mathrm{t}, J=7.4 \mathrm{~Hz}, 3$ H). ${ }^{13} \mathrm{C}\left\{{ }^{1} \mathrm{H}\right\} \mathrm{NMR}: \delta 209.4,134.1,130.0,68.8,50.4,31.1,25.4,13.5$. IR (neat, $\mathrm{cm}^{-1}$ ): 3426, 3029, 1711. Anal. Calcd (found) for $\mathrm{C}_{8} \mathrm{H}_{14} \mathrm{O}_{2}$ : C, 67.57 (67.24); H, 9.92 (10.07).

For $(\boldsymbol{E})-18:[19]$ 43\%. Enol:ketone $=13: 1$. TLC: $\mathrm{R}_{f}=0.38 .{ }^{1} \mathrm{H}$ NMR: $\delta 15.4$ (br s, $\left.1 \mathrm{H}\right), 6.89$ $(\mathrm{td}, J=6.6,15.6 \mathrm{~Hz}, 1 \mathrm{H}), 5.82(\mathrm{td}, J=1.8,15.6 \mathrm{~Hz}, 1 \mathrm{H}), 5.49(\mathrm{~s}, 1 \mathrm{H}), 2.29-2.20(\mathrm{~m}, 2 \mathrm{H}), 2.11(\mathrm{~s}, 3 \mathrm{H})$, $1.07(\mathrm{t}, J=7.4 \mathrm{~Hz}, 3 \mathrm{H}) .{ }^{13} \mathrm{C}\left\{{ }^{1} \mathrm{H}\right\} \mathrm{NMR}: \delta 197.2,178.7,146.4,125.1,100.0,27.0,26.1,12.8$. IR (neat, $\mathrm{cm}^{-1}$ ): 1722, 1651, 1589. Anal. Calcd. (found) for $\mathrm{C}_{8} \mathrm{H}_{12} \mathrm{O}_{2}$ : C, 68.54 (68.36); H, 8.63 (8.53).

\section{3-Butenyl $\beta$-Diketone Isotopomers}

6-Deuterio-7-octene-2,4-dione (3-6-d $)$. Isotopomer 3-6- $d_{1}$ was synthesized from 2,4pentanedione and 1-deuterio-2-propene-1-ol methanesulfonate employing a procedure analogous to that used to synthesize 8-nonene-2,4-dione[7] in 28\% yield. ${ }^{1} \mathrm{H}$ NMR: $\delta 5.79(\mathrm{ddd}, J=5.2,10.2,17.0 \mathrm{~Hz}, 1$ $\mathrm{H}), 2.36-2.26(\mathrm{~m}, 3 \mathrm{H}) .{ }^{13} \mathrm{C}\left\{{ }^{1} \mathrm{H}\right\} \mathrm{NMR}: \delta 137.1(\mathrm{~s}, \mathrm{IS}=68 \mathrm{ppb}), 37.7(\mathrm{~s}, \mathrm{IS}=72 \mathrm{ppb}), 29.4\left(\mathrm{t}, J_{\mathrm{CD}}=\right.$ $19.6 \mathrm{~Hz}, \mathrm{IS}=333 \mathrm{ppb})$.

(E)-7,8-dideuterio-7-octene-2,4-dione $\left[(\boldsymbol{E})-3-7,8-\boldsymbol{d}_{2}\right]$. 7-Octyne-2,4-dione $(\mathbf{S 4})$ was prepared employing a procedure similar to that used to synthesize methyl 3-oxo-6-heptynoate.[20] 2,4-Pentanedione $(3.1 \mathrm{~mL}, 30 \mathrm{mmol})$ was added dropwise to a suspension of $\mathrm{NaH}(0.77 \mathrm{~g}, 32 \mathrm{mmol})$ in $\mathrm{THF}(120 \mathrm{~mL})$ at 0 ${ }^{\circ} \mathrm{C}$ and stirred for $10 \mathrm{~min} . \mathrm{n}$-BuLi $(2.5 \mathrm{M}$ in hexanes, $8.0 \mathrm{~mL}, 20 \mathrm{mmol})$ was added slowly to the resulting solution at $-30{ }^{\circ} \mathrm{C}$ and stirred for $15 \mathrm{~min}$. Propargyl bromide ( $80 \%$ solution in toluene, $3.4 \mathrm{~mL}, 32 \mathrm{mmol}$ ) 
was added rapidly to the resulting pale yellow solution and stirred for $1 \mathrm{~h}$ at $-20{ }^{\circ} \mathrm{C}$. Following addition of saturated aqueous $\mathrm{NH}_{4} \mathrm{Cl}$, the reaction mixture was extracted with ether $(2 \times 50 \mathrm{~mL})$. The combined ether extracts were washed with brine, dried $\left(\mathrm{MgSO}_{4}\right)$, concentrated, and chromatographed (hexanes-ether $=$ $50: 1 \rightarrow 10: 1)$ to give $\mathbf{S 4}$ as a colorless oil $(2.20 \mathrm{~g}, 53 \%)$.

A suspension of Lindlar's catalyst (40 mg), quinoline (16 mg), and $\mathbf{S 4}$ (400 mg, $2.90 \mathrm{mmol})$ in acetone $(5 \mathrm{~mL})$ under $\mathrm{D}_{2}(1 \mathrm{~atm})$ was stirred vigorously for $1 \mathrm{~h}$, filtered through Celite, concentrated, and chromatographed (hexanes-ether $=100: 1)$ to give $(E)-3-7,8-d_{2}(280 \mathrm{mg}, 68 \%)$ as a colorless oil. ${ }^{1} \mathrm{H}$ NMR and MS analysis revealed formation of a $\sim 5: 1$ mixture of $(E)-3-7,8-d_{2}$ and 3-7,8,8- $d_{3}$; the presence of the $d_{3}$ isotopomer did not complicate stereochemical analysis of the products generated from $(E)-3-7,8-d_{2}$ (see below).

For S4: TLC: $\mathrm{R}_{f}=0.26 .{ }^{1} \mathrm{H}$ NMR (enol: ketone $\left.=\sim 3: 1\right): \delta 15.3($ br s, $1 \mathrm{H}), 5.51(\mathrm{~s}, 1 \mathrm{H}), 2.53-$ $2.42(\mathrm{~m}, 4 \mathrm{H}), 2.04(\mathrm{~s}, 3 \mathrm{H}), 1.96(\mathrm{t}, J=2.6 \mathrm{~Hz}, 1 \mathrm{H}) .{ }^{13} \mathrm{C}\left\{{ }^{1} \mathrm{H}\right\} \mathrm{NMR}: \delta 192.7,190.5,100.2,83.0,69.4$, 37.5, 24.9, 14.7. IR (neat, $\mathrm{cm}^{-1}$ ): 3293, 2119, 1730, 1706, 1615. Anal. calcd (found) for $\mathrm{C}_{8} \mathrm{H}_{10} \mathrm{O}_{2}: \mathrm{C}_{\text {, }}$ 69.54 (69.68); H, 7.30 (7.24).

For $(\boldsymbol{E})-3-7,8-d_{2}:$ This sample consisted of a $\sim 5: 1$ mixture of $(E)-3-7,8-d_{2}$ and 3-7,8,8- $d_{3} .{ }^{1} \mathrm{H}$ NMR: $\delta 5.04$ (br s, $\left.1 \mathrm{H},-\mathrm{CH}_{2} \mathrm{CD}=\mathrm{C} H \mathrm{D}\right) .{ }^{13} \mathrm{C}\left\{{ }^{1} \mathrm{H}\right\} \mathrm{NMR}: \delta 136.7\left(\mathrm{t}, J_{\mathrm{CD}}=23 \mathrm{~Hz}, \mathrm{IS}=447 \mathrm{ppb}\right.$, $\left.-\mathrm{CH}_{2} C \mathrm{D}=\mathrm{CHD}\right), 136.6\left(\mathrm{t}, J_{\mathrm{CD}}=23 \mathrm{~Hz}, \mathrm{IS}=526 \mathrm{ppb},-\mathrm{CH}_{2} C \mathrm{D}=\mathrm{CD}_{2}\right), 115.4\left(\mathrm{t}, J_{\mathrm{CD}}=24 \mathrm{~Hz}, \mathrm{IS}=413\right.$ ppb, $-\mathrm{CH}_{2} \mathrm{CD}=\mathrm{CHD}$ ), 29.5 (s, IS = $159 \mathrm{ppb},-\mathrm{CH}_{2} \mathrm{CD}=\mathrm{CHD}$ ). The quintet corresponding to $-\mathrm{CH}_{2} \mathrm{CD}=C \mathrm{D}_{2}$ was not resolved from the triplet at $\delta 115.4$.

(Z)-7,8-dideuterio-7-octene-2,4-dione $\left[(\boldsymbol{Z})-3-7,8-d_{2}\right]$. Reaction of 2,4-pentanedione and $\mathbf{S 2}$ that contained $\sim 25 \%$ 3-bromo-1,1,2-trideuteriopropene employing a procedure analogous to that used to synthesize 8-nonene-2,4-dione, ${ }^{[7]}$ gave $(Z)-3-7,8-d_{2}$ that contained $\sim 25 \%$ 3-7,8,8- $d_{3}$ by ${ }^{1} \mathrm{H}$ NMR and MS analysis in $56 \%$ yield; the presence of the $d_{3}$ isotopomer did not complicate stereochemical analysis of the products generated from $(Z)-3-7,8-d_{2}$ (see below). ${ }^{1} \mathrm{H}$ NMR: $\delta 4.98$ (br s, $\left.1 \mathrm{H},-\mathrm{CH}_{2} \mathrm{CD}=\mathrm{CHD}\right)$. ${ }^{13} \mathrm{C}\left\{{ }^{1} \mathrm{H}\right\} \mathrm{NMR}: \delta 136.7\left(\mathrm{t}, J_{\mathrm{CD}}=23 \mathrm{~Hz}, \mathrm{IS}=451 \mathrm{ppb},-\mathrm{CH}_{2} C \mathrm{D}=\mathrm{CHD}\right), 136.6\left(\mathrm{t}, J_{\mathrm{CD}}=23 \mathrm{~Hz}, \mathrm{IS}=\right.$ $\left.564 \mathrm{ppb},-\mathrm{CH}_{2} \mathrm{CD}=\mathrm{CD}_{2}\right), 115.3\left(\mathrm{t}, J_{\mathrm{CD}}=24 \mathrm{~Hz}, \mathrm{IS}=463 \mathrm{ppb},-\mathrm{CH}_{2} \mathrm{CD}=C \mathrm{HD}\right), 29.5(\mathrm{~s}, \mathrm{IS}=167 \mathrm{ppb}$ 
$\left.-\mathrm{CH}_{2} \mathrm{CD}=\mathrm{CHD}\right)$. The quintet corresponding to $-\mathrm{CH}_{2} \mathrm{CD}=\mathrm{CD}_{2}$ was not resolved from the triplet at $\delta$ 115.3.

3,3-Dideuterio-7-octene-2,4-dione (3-3,3- $\left.\boldsymbol{d}_{2}\right)$. Alkenyl dione 3-3,3- $d_{2}$ was prepared from 3 and $\mathrm{D}_{2} \mathrm{O}$ in $87 \%$ yield employing a procedure analogous to that used to synthesize 3,3-dideuterio-2,4pentanedione. [21] ${ }^{1} \mathrm{H}$ NMR analysis of $3-3,3-d_{2}$ revealed $\sim 75 \%$ deuteration of the $\mathrm{C}(3)$ position with no detectable $(\leq 5 \%)$ accumulation of deuterium at the $\mathrm{C}(1)$ and $\mathrm{C}(5)$ positions. The presence of 3 did not complicate analysis of the cyclization of $3-3,3-d_{2} .{ }^{13} \mathrm{C}\left\{{ }^{1} \mathrm{H}\right\} \mathrm{NMR}: \delta 99.8\left(\mathrm{t}, J_{\mathrm{CD}}=25.2 \mathrm{~Hz}, \mathrm{IS}=375\right.$ $\left.\mathrm{ppb},-\mathrm{COCD}_{2} \mathrm{CO}-\right)$.

8,8-Dideuterio-7-octene-2,4-dione (3-8,8- $\left.\boldsymbol{d}_{\mathbf{2}}\right)$. Alkenyl dione 1-8,8- $\boldsymbol{d}_{2}$ was synthesized from 2,4-pentanedione and $\mathbf{S 1}$ in $32 \%$ yield employing a procedure analogous to that used to synthesize 8nonene-2,4-dione. [7] ${ }^{1} \mathrm{H}$ NMR analysis revealed complete $(\geq 95 \%)$ deuteration of the $\mathrm{C}(8)$ position. ${ }^{1} \mathrm{H}$ NMR: $\delta 5.79\left(\right.$ br s, $\left.1 \mathrm{H},-\mathrm{CH}_{2} \mathrm{CH}=\mathrm{CD}_{2}\right) .{ }^{13} \mathrm{C}\left\{{ }^{1} \mathrm{H}\right\} \mathrm{NMR}: \delta 136.9\left(\mathrm{~s}, \mathrm{IS}=212 \mathrm{ppb},-\mathrm{CH}_{2} C \mathrm{H}=\mathrm{CD}_{2}\right)$, 115.3 (quintet, $J_{\mathrm{CD}}=24 \mathrm{~Hz}, \mathrm{IS}=645 \mathrm{ppb},-\mathrm{CH}_{2} \mathrm{CH}=\mathrm{CD}_{2}$ ).

1,1,1,5,5-Pentadeuterio-7-octene-2,4-dione (3-1,1,1,5,5- $\left.d_{5}\right)$. Alkenyl dione 3 was converted to 1,1,1,3,3,5,5-heptadeuterio-7-octene-2,4-dione (3-1,1,1,3,3,5,5- $\left.d_{7}\right)$ employing a procedure analogous to that used to synthesize 1,1,1,3,3,5,5,5-octadeuterio-2,4-pentanedione.[21] Compound 3-1,1,1,3,3,5,5- $d 7$ was then flushed through a short column of silica gel to give $3-1,1,1,5,5-d_{5}$ in $10 \%$ yield from 3 . ${ }^{1} \mathrm{H}$ and ${ }^{13} \mathrm{C}$ NMR analysis of 1-1,1,1,5,5- $d_{5}$ revealed complete ( $\left.\geq 95 \%\right)$ deuteration of the acyl methyl group and $C(5)$ methylene group with no detectable deuteration $(\leq 5 \%)$ of the $\mathrm{C}(3)$ methylene group. ${ }^{1} \mathrm{H}$ NMR: $\delta 2.34(\mathrm{~d}$, $J=6.8 \mathrm{~Hz}, 2 \mathrm{H},-\mathrm{CD}_{2} \mathrm{CH}_{2} \mathrm{CH}=\mathrm{CH}_{2}$ ). ${ }^{13} \mathrm{C}\left\{{ }^{1} \mathrm{H}\right\} \mathrm{NMR}: \delta 37.2$ (quintet, $J_{\mathrm{CD}}=20 \mathrm{~Hz}, \mathrm{IS}=645 \mathrm{ppb}$, $-\mathrm{CD}_{2} \mathrm{CH}_{2} \mathrm{CH}=\mathrm{CH}_{2}$ ), 29.6 (s, IS = $\left.100 \mathrm{ppb},-\mathrm{CD}_{2} \mathrm{CH}_{2} \mathrm{CH}=\mathrm{CH}_{2}\right), 24.4\left(\mathrm{~m},-\mathrm{COCD}_{3}\right)$.

6,6-Dideuterio-7-octene-2,4-dione (3-6,6- $\left.\boldsymbol{d}_{2}\right)$. Alkenyl diketone 3-6,6- $d_{2}$ was synthesized from 2,4-pentanedione and $\mathbf{S 3}$ in $28 \%$ yield employing a procedure analogous to that used to synthesize 8nonene-2,4-dione. [7] ${ }^{1} \mathrm{H}$ and ${ }^{13} \mathrm{C}$ NMR analysis of 3-6,6- $d_{2}$ revealed complete $(\geq 95 \%)$ deuteration of the $\mathrm{C}(6)$ position. ${ }^{1} \mathrm{H}$ NMR: $\delta 5.80\left(\mathrm{dd}, J=10.0,17.2 \mathrm{~Hz}, 1 \mathrm{H},-\mathrm{CH}_{2} \mathrm{CD}_{2} \mathrm{CH}=\mathrm{CH}_{2}\right), 2.35$ (br s, $2 \mathrm{H}$, $\left.-\mathrm{CH}_{2} \mathrm{CD}_{2} \mathrm{CH}=\mathrm{CH}_{2}\right) .{ }^{13} \mathrm{C}\left\{{ }^{1} \mathrm{H}\right\} \mathrm{NMR}: \delta 137.1\left(\mathrm{~s}, \mathrm{IS}=129 \mathrm{ppb},-\mathrm{CH}_{2} \mathrm{CD}_{2} \mathrm{CH}=\mathrm{CH}_{2}\right), 37.7(\mathrm{~s}, \mathrm{IS}=144$ ppb, $-\mathrm{CH}_{2} \mathrm{CD}_{2} \mathrm{CH}=\mathrm{CH}_{2}$ ), 29.1 (quintet, $J=20 \mathrm{~Hz}, \mathrm{IS}=661 \mathrm{ppb},-\mathrm{CH}_{2} \mathrm{CD}_{2} \mathrm{CH}=\mathrm{CH}_{2}$ ). 


\section{Syntheses of Palladium(II) Catalysts}

$\mathbf{P d C l}_{2}\left(\mathrm{NCCH}_{2} \mathbf{C H}_{3}\right)_{2}$. A suspension of $\mathrm{PdCl}_{2}(500 \mathrm{mg}, 2.82 \mathrm{mmol})$ in propionitrile $(10 \mathrm{~mL})$ was stirred at room temperature for 2 days, poured into hexanes $(100 \mathrm{~mL})$, filtered, washed with hexanes, and dried under vacuum. A pale yellow solid was obtained (760 mg, 94\%). ${ }^{1} \mathrm{H}$ NMR: $\delta 2.66$ (q, $J=7.6$ $\mathrm{Hz}, 4 \mathrm{H}), 1.33(\mathrm{t}, J=7.6 \mathrm{~Hz}, 6 \mathrm{H})$. Anal. calcd (found) for $\mathrm{C}_{6} \mathrm{H}_{10} \mathrm{Cl}_{2} \mathrm{~N}_{2} \mathrm{Pd}: \mathrm{C}, 25.07$ (24.79); H, 3.51 (3.23); N, 9.74 (9.39).

$\mathbf{P d C l}_{2}\left(\mathrm{NCCH}_{2} \mathrm{CH}_{2} \mathrm{CH}_{3}\right)_{2}$. Bis(butyronitrile)palladium dichloride was isolated as a yellow solid (850 $\mathrm{mg}, 97 \%)$ from the reaction of $\mathrm{PdCl}_{2}(500 \mathrm{mg}, 2.82 \mathrm{mmol})$ in butyronitrile $(10 \mathrm{~mL})$ at room temperature for 2 days, employing a procedure similar to that used to synthesize $\mathrm{PdCl}_{2}(\mathrm{EtCN})_{2} .{ }^{1} \mathrm{H} \mathrm{NMR}$ : $\delta 2.62(\mathrm{t}, J=6.8 \mathrm{~Hz}, 4 \mathrm{H}), 1.78-1.69(\mathrm{~m}, 4 \mathrm{H}), 1.06(\mathrm{t}, J=7.4 \mathrm{~Hz}, 6 \mathrm{H})$. Anal. calcd (found) for $\mathrm{C}_{8} \mathrm{H}_{14} \mathrm{Cl}_{2} \mathrm{~N}_{2} \mathrm{Pd}: \mathrm{C}, 30.45$ (30.10); H, 4.47 (4.28); N, 8.88 (8.81).

$\mathbf{P d C l}_{2}\left[\mathbf{N C C H}\left(\mathrm{CH}_{3}\right)_{2}\right]_{2}$. Bis(isobutyronitrile)palladium dichloride was isolated as a reddish orange solid $(800 \mathrm{mg}, 92 \%)$ from the reaction of $\mathrm{PdCl}_{2}(500 \mathrm{mg}, 2.82 \mathrm{mmol})$ in isobutyronitrile $(10 \mathrm{~mL})$ at room temperature for 2 days, employing a procedure similar to that used to synthesize $\mathrm{PdCl}_{2}(\mathrm{EtCN})_{2} .{ }^{1} \mathrm{H}$ NMR: $\delta 2.97$ (septet, $J=6.8 \mathrm{~Hz}, 2 \mathrm{H}$ ), $1.35(\mathrm{~d}, J=6.8 \mathrm{~Hz}, 12 \mathrm{H}$ ). Anal. calcd (found) for $\mathrm{C}_{8} \mathrm{H}_{14} \mathrm{Cl}_{2} \mathrm{~N}_{2} \mathrm{Pd}: \mathrm{C}, 30.45$ (30.43); H, 4.47 (4.22); N, 8.88 (8.49).

$\mathbf{P d C l}_{2}\left[\mathbf{N C}-3,5-\mathbf{C}_{6} \mathbf{H}_{3}\left(\mathrm{CF}_{3}\right)_{2}\right]_{2}$. Bis(3,5-bistrifluoromethylbenzonitrile) palladium dichloride was isolated as a yellow solid $(560 \mathrm{mg}, 100 \%)$ from the reaction of $\mathrm{PdCl}_{2}(150 \mathrm{mg}, 0.85 \mathrm{mmol}) 3,5-$ bistrifluoromethylbenzonitrile $(5.0 \mathrm{~g}, 21 \mathrm{mmol})$ at room temperature for $32 \mathrm{~h}$, employing a procedure similar to that used to synthesize $\mathrm{PdCl}_{2}(\mathrm{EtCN})_{2}$. ${ }^{1} \mathrm{H}$ NMR: $\delta 8.11$ (broad singlet, $6 \mathrm{H}$ ). Anal. calcd (found) for $\mathrm{C}_{18} \mathrm{H}_{6} \mathrm{~N}_{2} \mathrm{Cl}_{2} \mathrm{~F}_{12} \mathrm{Pd}$ : C, 32.98 (32.70); H, 0.92 (0.88); N, 4.27 (4.43).

\section{Cyclohexanones}

2-Propionylcyclohexanone (Table 2, entry 3). ${ }^{[22] ~ E n o l: d i o n e ~} \geq 15: 1 . \quad$ TLC: $\mathrm{R}_{f}=0.59 .{ }^{1} \mathrm{H}$ NMR: $\delta 2.43(\mathrm{q}, J=7.2 \mathrm{~Hz}, 2 \mathrm{H}), 2.33-2.29(\mathrm{~m}, 4 \mathrm{H}), 1.69-1.66(\mathrm{~m}, 4 \mathrm{H}), 1.10(\mathrm{t}, J=7.2 \mathrm{~Hz}, 3 \mathrm{H})$. ${ }^{13} \mathrm{C}\left\{{ }^{1} \mathrm{H}\right\}$ NMR: $\delta$ 203.2, 180.6, 106.9, 31.1, 30.7, 24.0, 23.1, 22.0, 8.5. 
2-(2',2'-Dimethylpropionyl)cyclohexanone (Table 2, entry 4). ${ }^{23]}$ Enol:dione $\geq 15: 1$. TLC: $\mathrm{R}_{f}=0.41 .{ }^{1} \mathrm{H}$ NMR: $\delta$ 3.95-3.92 (m, $\left.1 \mathrm{H}\right), 2.55-2.48(\mathrm{~m}, 1 \mathrm{H}), 2.39-2.31(\mathrm{~m}, 1 \mathrm{H}), 2.12-2.03(\mathrm{~m}, 1 \mathrm{H})$, 2.00-1.90 (m, $3 \mathrm{H}), 1.87-1.81(\mathrm{~m}, 1 \mathrm{H}), 1.70-1.62(\mathrm{~m}, 1 \mathrm{H}), 1.11(\mathrm{~s}, 9 \mathrm{H}) .{ }^{13} \mathrm{C}\left\{{ }^{1} \mathrm{H}\right\}$ NMR: $\delta$ 212.7, 208.5, 57.8, 45.4, 42.2, 31.3, 27.4, 26.0, 23.6.

2-Acetyl-2-methylcyclohexanone (Table 2, entry 5).[24] $\mathrm{TLC}: \mathrm{R}_{f}=0.45 .{ }^{1} \mathrm{H}$ NMR: $\delta$ 2.492.46 (m, $1 \mathrm{H}), 2.45-2.43$ (m, $1 \mathrm{H}), 2.34-2.26(\mathrm{~m}, 1 \mathrm{H}), 2.10$ (s, $3 \mathrm{H}), 2.01-1.94(\mathrm{~m}, 1 \mathrm{H}), 1.75-1.62$ (m, 3 H), 1.49-1.46 (m, $1 \mathrm{H}), 1.24(\mathrm{~s}, 3 \mathrm{H}) .{ }^{13} \mathrm{C}\left\{{ }^{1} \mathrm{H}\right\} \mathrm{NMR}: \delta$ 210.9, 207.9, 64.2, 41.6, 37.1, 27.6, 26.0, 22.6, 21.1.

2-Acetyl-2-benzylcyclohexanone (Table 2, entry 6). TLC: $\mathrm{R}_{f}=0.42 .{ }^{1} \mathrm{H}$ NMR: $\delta$ 7.24-7.20 (m, $3 \mathrm{H}), 7.08-7.05(\mathrm{~m}, 2 \mathrm{H}), 3.16,3.08(\mathrm{ABq}, J=6.0 \mathrm{~Hz}, 2 \mathrm{H}), 2.54-2.48(\mathrm{~m}, 1 \mathrm{H}), 2.39-2.33(\mathrm{~m}, 1 \mathrm{H})$, 2.28-2.20 (m, 1 H), 2.09 (s, 3 H), 1.99-1.95 (m, 1 H), 1.73-1.70 (m, 1 H), 1.64-1.58 (m, 2 H), 1.46-1.39 (m, $1 \mathrm{H}) .{ }^{13} \mathrm{C}\left\{{ }^{1} \mathrm{H}\right\}$ NMR: $\delta$ 209.8, 206.1, 136.5, 130.6, 128.5, 127.1, 69.0, 42.4, 40.3, 34.3, 27.3, 27.2, 22.5. IR (neat, $\mathrm{cm}^{-1}$ ): 3085, 3061, 3028, 2941, 2866, 1956, 1881, 1811, 1713, 1693, 1603, 1495, 1453 , 1434, 1358, 1311. Anal. calcd (found) for $\mathrm{C}_{15} \mathrm{H}_{18} \mathrm{O}_{2}$ : C, 78.23 (78.13); $\mathrm{H}, 7.88$ (7.80).

2-Acetyl-3-methylcyclohexanone (Table 2, entry 7 and 8).[25] Enol:dione $\geq 15: 1$. TLC: $\mathrm{R}_{f}=$ 0.49. ${ }^{1} \mathrm{H}$ NMR: $\delta$ 2.77-2.73 (m, $\left.1 \mathrm{H}\right), 2.35-2.31(\mathrm{~m}, 2 \mathrm{H}), 2.18(\mathrm{~s}, 3 \mathrm{H}), 1.84-1.75(\mathrm{~m}, 1 \mathrm{H}), 1.72-1.60$ $(\mathrm{m}, 3 \mathrm{H}), 1.10(\mathrm{~d}, J=6.8 \mathrm{~Hz}, 3 \mathrm{H}) .{ }^{13} \mathrm{C}\left\{{ }^{1} \mathrm{H}\right\} \mathrm{NMR}: \delta 198.3,184.4,113.2,31.7,30.3,28.2,24.2,22.0$, 17.0.

2-Acetyl-3-butylcyclohexanone (Table 2, entry 9). ${ }^{[26]}$ Enol:dione $\geq 15: 1$. TLC: $R_{f}=0.44$. ${ }^{1} \mathrm{H}$ NMR: $\delta$ 2.50-2.46 (m, $\left.1 \mathrm{H}\right), 2.34-2.31(\mathrm{~m}, 2 \mathrm{H}), 2.15(\mathrm{~s}, 3 \mathrm{H}), 1.83-1.62(\mathrm{~m}, 3 \mathrm{H}), 1.55-1.50(\mathrm{~m}, 1 \mathrm{H})$, 1.42-1.20 (m, $6 \mathrm{H}), 0.90(\mathrm{t}, J=7.0 \mathrm{~Hz}, 3 \mathrm{H}) .{ }^{13} \mathrm{C}\left\{{ }^{1} \mathrm{H}\right\}$ NMR: $\delta$ 198.0, 184.4, 113.3, 35.1, 33.5, 31.5, $30.4,25.6,24.2,23.1,16.9,14.4$.

2-Acetyl-3-phenylcyclohexanone (Table 2, entry 10).[27] Enol:dione $\geq 15: 1$. TLC: $\mathrm{R}_{f}=0.12$. ${ }^{1} \mathrm{H}$ NMR: $\delta$ 7.33-7.29 (m, $\left.2 \mathrm{H}\right), 7.23-7.16(\mathrm{~m}, 3 \mathrm{H}), 3.93(\mathrm{dd}, J=3.2,5.6 \mathrm{~Hz}, 1 \mathrm{H}), 2.45-2.42(\mathrm{~m}, 2 \mathrm{H})$, 2.07-1.94 (m, $1 \mathrm{H}), 1.85(\mathrm{~s}, 3 \mathrm{H}), 1.84-1.80(\mathrm{~m}, 1 \mathrm{H}), 1.60-1.53(\mathrm{~m}, 2 \mathrm{H}) .{ }^{13} \mathrm{C}\left\{{ }^{1} \mathrm{H}\right\}$ NMR: $\delta 201.4$, $183.1,145.7,128.8,128.2,126.6,109.9,40.5,32.4,31.2,25.7,16.9$. 
2-Acetyl-3,3-dimethylcyclohexanone (Table 2, entry 11). ${ }^{[28]}$ Enol:dione $\leq 1: 15$. TLC: $\mathbf{R}_{f}=$ 0.34. ${ }^{1} \mathrm{H}$ NMR: $\delta 3.44$ (s, $\left.1 \mathrm{H}\right), 2.57$ (ddd, $\left.J=6.4,10.0,16.4 \mathrm{~Hz}, 1 \mathrm{H}\right), 2.31-2.25(\mathrm{~m}, 1 \mathrm{H}), 2.17(\mathrm{~s}, 3 \mathrm{H})$, 2.04 (ddd, $J=4.4,10.4,14.8 \mathrm{~Hz}, 1 \mathrm{H}), 1.95-1.89$ (m, $1 \mathrm{H}), 1.85-1.77$ (m, $1 \mathrm{H}), 1.41-1.35$ (m, $1 \mathrm{H}), 1.04$ (s, $3 \mathrm{H}), 0.95$ (s, $3 \mathrm{H}) .{ }^{13} \mathrm{C}\left\{{ }^{1} \mathrm{H}\right\}$ NMR: $\delta$ 208.1, 205.3, 75.1, 39.6, 39.5, 35.3, 33.9, 28.3, 26.4, 22.2.

2-Ethoxycarbonylcyclohexanone (Table 2, entry 12). ${ }^{[29]}$ Enol:dione $=3: 1$. TLC: $\mathrm{R}_{f}=0.59$. ${ }^{1} \mathrm{H}$ NMR: $\delta 12.24$ (s, $\left.1 \mathrm{H}\right), 4.20$ (q, $\left.J=7.2 \mathrm{~Hz}, 2 \mathrm{H}\right), 2.27-2.20$ (m, $\left.4 \mathrm{H}\right), 1.70-1.64$ (m, $\left.2 \mathrm{H}\right), 1.62-1.58$ $(\mathrm{m}, 2 \mathrm{H}), 1.29(\mathrm{t}, J=7.2 \mathrm{~Hz}, 3 \mathrm{H}) .{ }^{13} \mathrm{C}\left\{{ }^{1} \mathrm{H}\right\}$ NMR: $\delta 173.1,172.3,98.1,60.5,29.4,22.8,22.7,22.3$, 14.6 .

Cyclization of Methyl 3-Oxo-6-heptenoate (Table 2, entry 13). A solution of 4 (17 mg, 0.06 $\mathrm{mmol})$ and methyl 3-oxo-6-heptenoate $(100 \mathrm{mg}, 0.64 \mathrm{mmol})$ in dioxane $(25 \mathrm{~mL})$ was stirred at room temperature for $24 \mathrm{~h}$, concentrated, and chromatographed (hexanes-EtOAc $=125: 1$ ) to give two fractions. The first eluting fraction consisted of pure 2-methoxycarbonylcyclohexanone (21 $\mathrm{mg}, 21 \%)$ as a pale yellow oil. The second fraction consisted of a 1:1:3 mixture of methyl 3-oxo-6-heptenoate, $(Z)$-methyl 3oxo-5-heptenoate, and (E)-methyl 3-oxo-5-heptenoate $(51 \mathrm{mg}, 55 \%)$ as a colorless oil. The methyl 3-oxo5-heptenoate isomers were characterized via ${ }^{1} \mathrm{H}$ and ${ }^{13} \mathrm{C}$ NMR analysis of the mixture and by comparison to the NMR spectra of pure methyl 3-oxo-6-heptenoate.

For 2-methoxycarbonylcyclohexanone. TLC: $\mathrm{R}_{f}=0.67 .{ }^{1} \mathrm{H}$ NMR (enol tautomer): $\delta 12.19$ (s, $1 \mathrm{H}), 3.75$ (s, $3 \mathrm{H}), 2.27$ (t, $J=6.4 \mathrm{~Hz}, 2 \mathrm{H}), 2.21$ (t, $J=6.0 \mathrm{~Hz}, 2 \mathrm{H}), 1.69-1.66(\mathrm{~m}, 2 \mathrm{H}), 1.62-1.58(\mathrm{~m}$, $2 \mathrm{H}) .{ }^{13} \mathrm{C}\left\{{ }^{1} \mathrm{H}\right\}$ NMR (enol tautomer): $\delta$ 173.4, 172.5, 98.0, 51.7, 29.4, 22.8, 22.7, 22.2.

For $(\boldsymbol{E})$-methyl 3-oxo-5-heptenoate: ${ }^{1} \mathrm{H}$ NMR (partial): $\delta 3.72$ (s, $\left.3 \mathrm{H}\right), 3.46(\mathrm{~s}, 2 \mathrm{H}), 3.20$ (br d, $J=6.8 \mathrm{~Hz}, 2 \mathrm{H}), 1.69(\mathrm{br} \mathrm{d}, J=5.6 \mathrm{~Hz}, 3 \mathrm{H}) .{ }^{13} \mathrm{C}\left\{{ }^{1} \mathrm{H}\right\}$ NMR: $\delta$ 201.6, 167.9, 131.2, 122.3, 52.6, 48.5, 47.1, 18.3.

For (Z)-methyl 3-oxo-5-heptenoate: ${ }^{1} \mathrm{H}$ NMR (partial): $\delta 3.72$ (s, $\left.3 \mathrm{H}\right), 3.47$ (s, $\left.2 \mathrm{H}\right), 3.29$ (br d, $J=7.2 \mathrm{~Hz}, 2 \mathrm{H}), 1.63($ br d, $J=6.8 \mathrm{~Hz}, 3 \mathrm{H}) .{ }^{13} \mathrm{C}\left\{{ }^{1} \mathrm{H}\right\}$ NMR: $\delta$ 201.0, 167.9, 129.1, 121.1, 52.6, 49.4, 48.7, 13.4 .

2-Benzoxycarbonylcyclohexanone (Table 2, entry 14). ${ }^{[30]}$ Enol:dione $=4: 1$. TLC: $\mathrm{R}_{f}=0.66$. ${ }^{1} \mathrm{H}$ NMR: $\delta 12.16(\mathrm{~s}, 1 \mathrm{H}), 7.38-7.31(\mathrm{~m}, 5 \mathrm{H}), 5.21(\mathrm{~s}, 2 \mathrm{H}), 2.30-2.27(\mathrm{~m}, 4 \mathrm{H}), 1.70-1.66(\mathrm{~m}, 2 \mathrm{H})$, 
1.64-1.58 (m, $2 \mathrm{H}) .{ }^{13} \mathrm{C}\left\{{ }^{1} \mathrm{H}\right\}$ NMR: $\delta$ 172.9, 172.8, 136.5, 128.9, 128.4, 128.2, 98.0, 66.0, 29.5, 22.7, 22.6, 22.2.

Figure S1. Concentration versus time plot for the reaction of $3\left([3]_{0}=250 \mathrm{mM}\right)$ with $4\left([4]_{0}=25 \mathrm{mM}\right)$ in dioxane at room temperature.

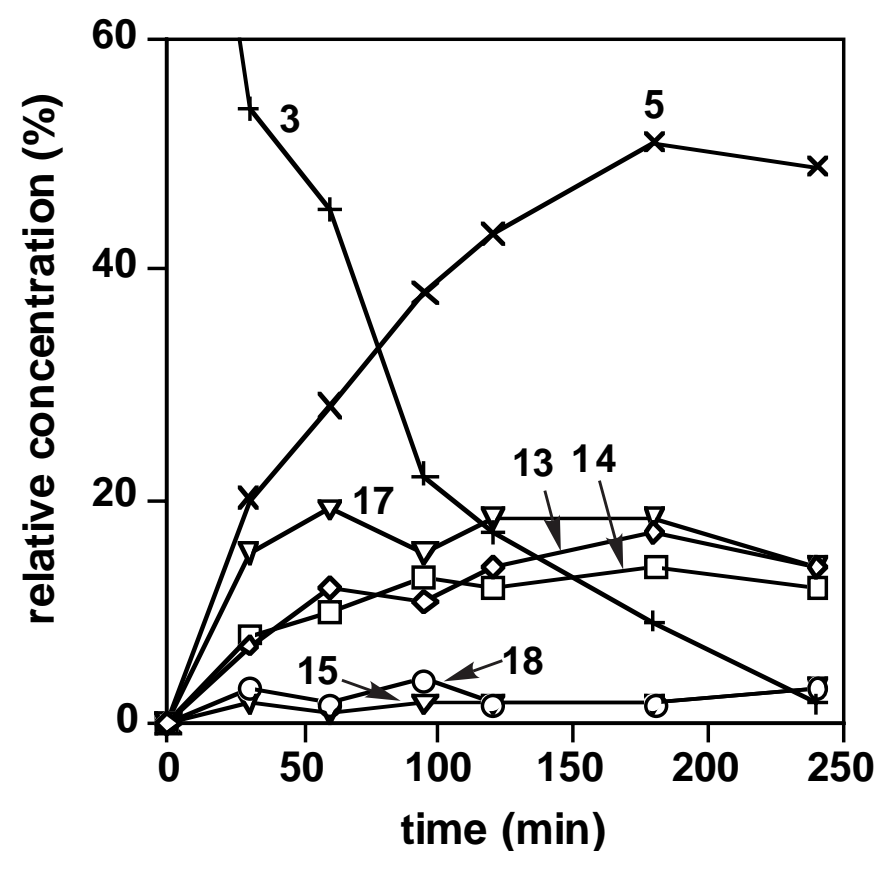


Figure S2. Aliphatic region of the ${ }^{13} \mathrm{C}\left\{{ }^{1} \mathrm{H}\right\}$ NMR spectrum of 2-acetylcyclohexanone (5).

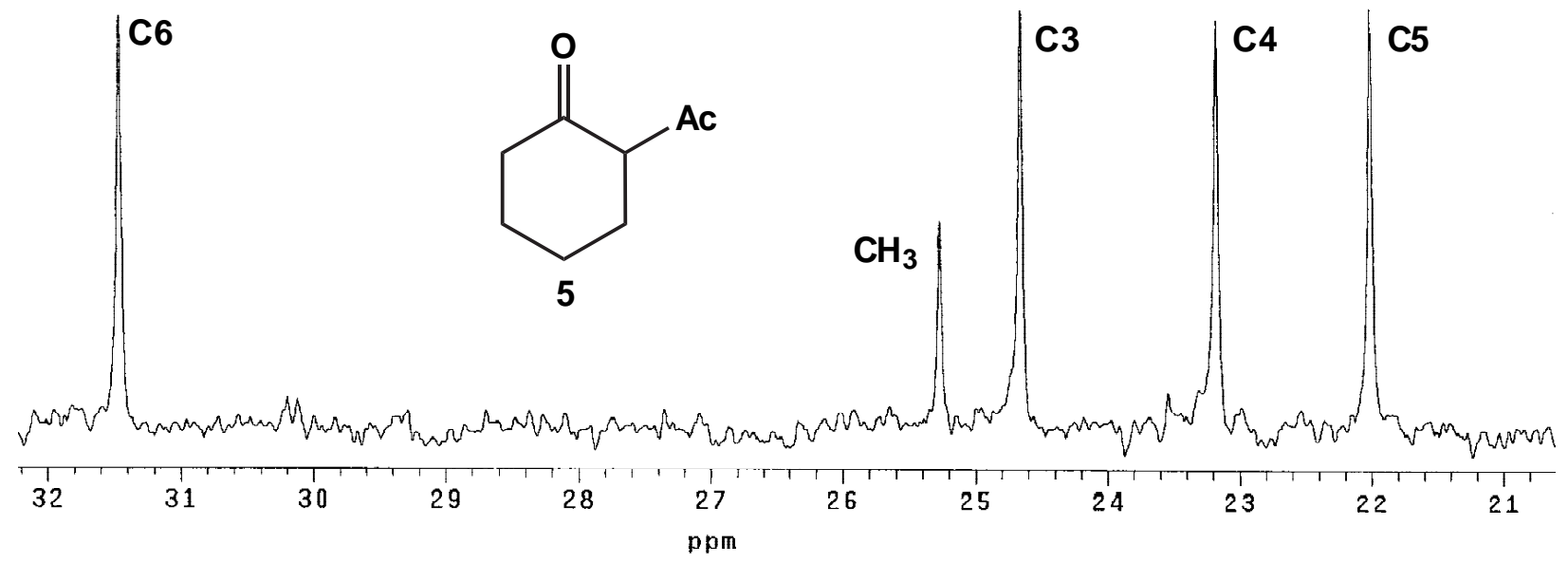

Figure S3. Aliphatic region of the ${ }^{1} \mathrm{H}_{-}{ }^{13} \mathrm{C}$ COSY NMR spectrum of 5.

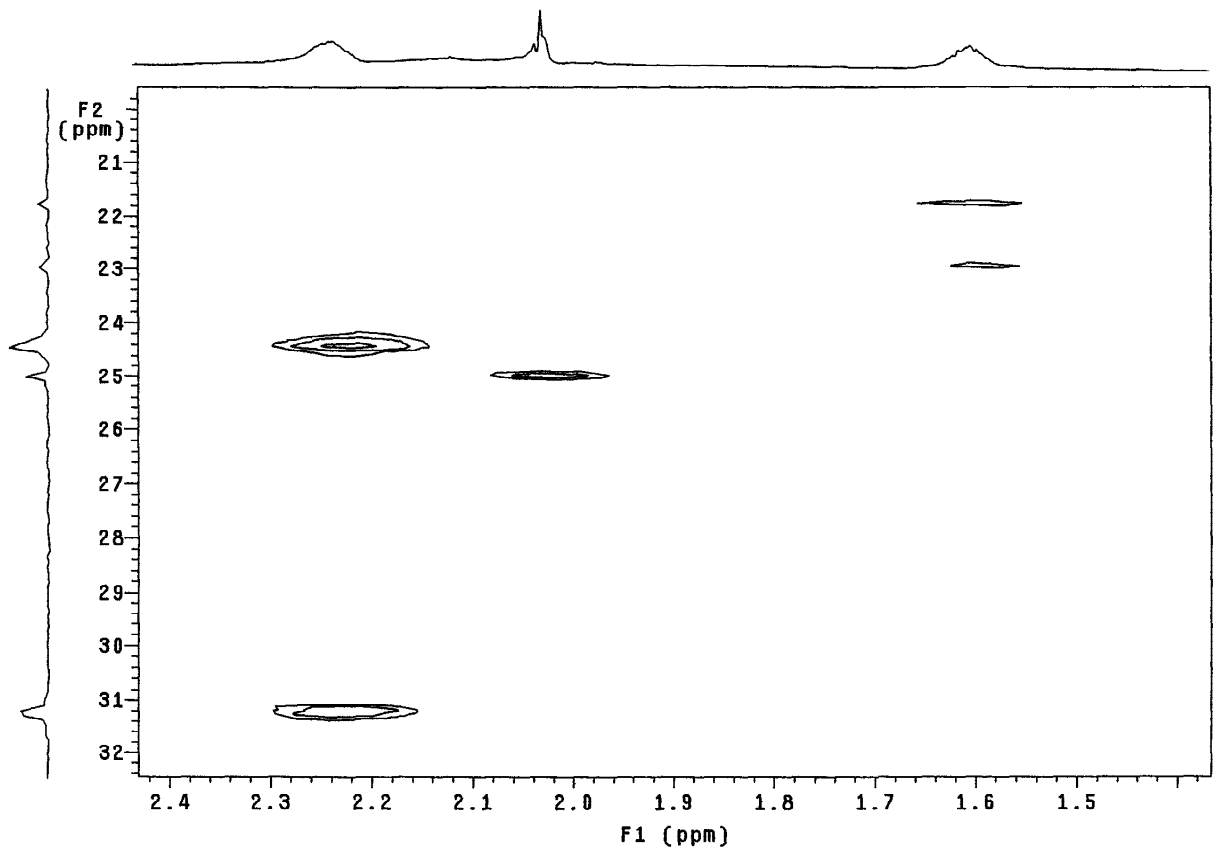


Figure S4. Aliphatic region of the ${ }^{13} \mathrm{C}\left\{{ }^{1} \mathrm{H}\right\}$ NMR spectrum of an $\sim 5: 1$ mixture of $c i s-5-3,4-d_{2}$ and 5$3,3,4-d_{3}$.

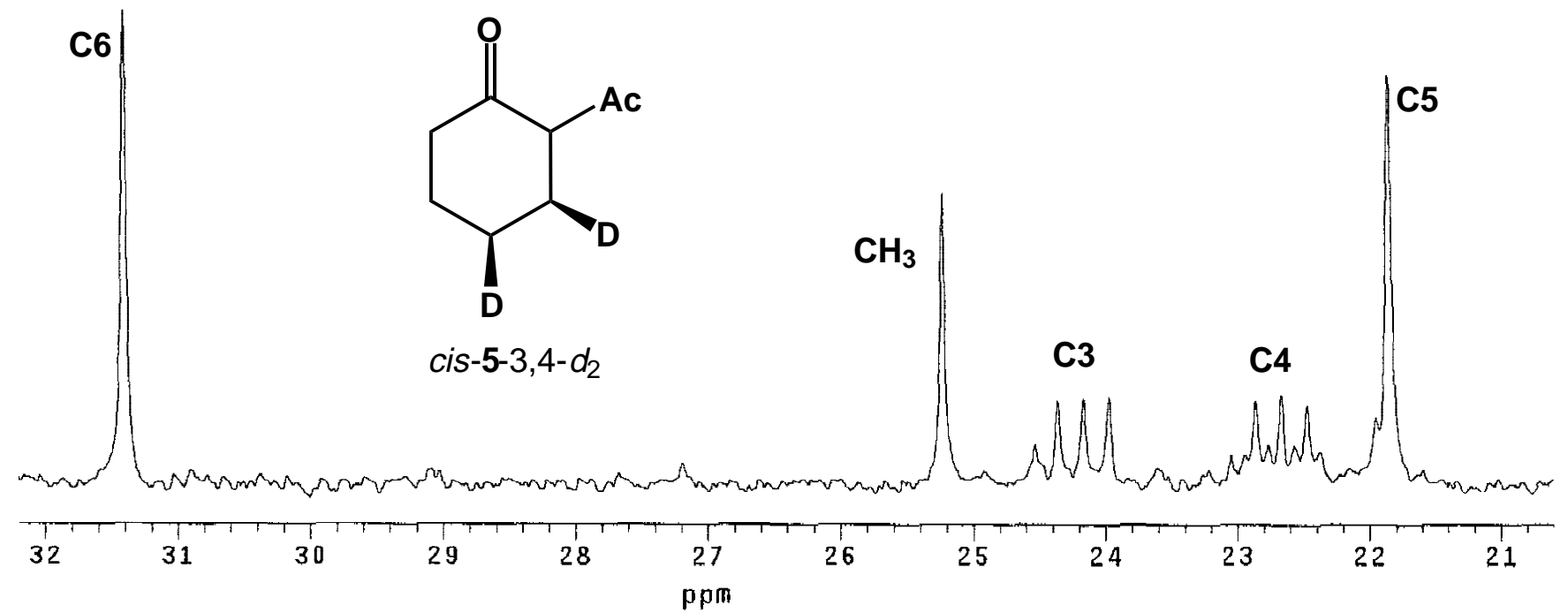

Figure S5. Aliphatic region of the ${ }^{13} \mathrm{C}\left\{{ }^{1} \mathrm{H}\right\}$ NMR spectrum of a $\sim 3: 1$ mixture of trans-5-3,4- $d_{2}$ and 5$3,3,4-d_{3}$.

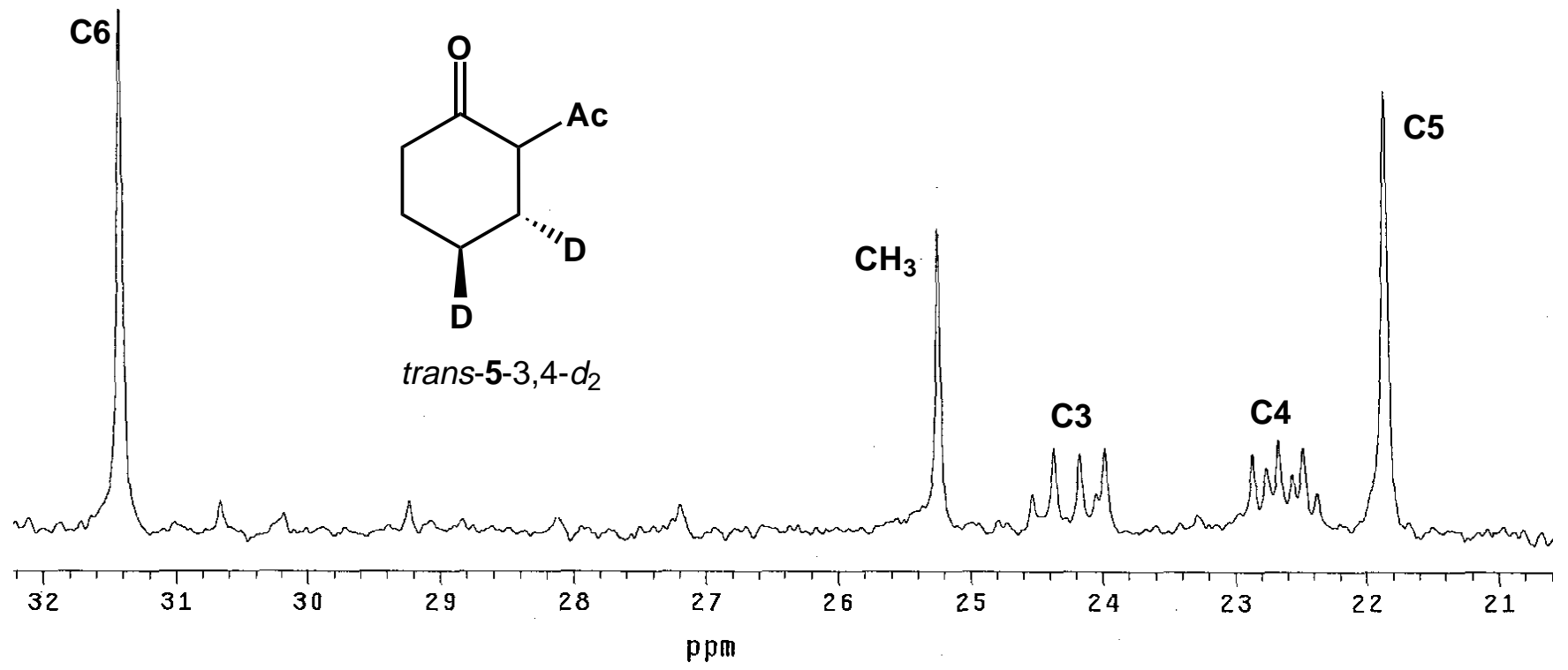


Figure S6. Aliphatic region of the ${ }^{13} \mathrm{C}\left\{{ }^{1} \mathrm{H}\right\}$ NMR spectrum of a $\sim 3: 1$ mixture of 5-6- $d_{1}$ and $\mathbf{5}$.

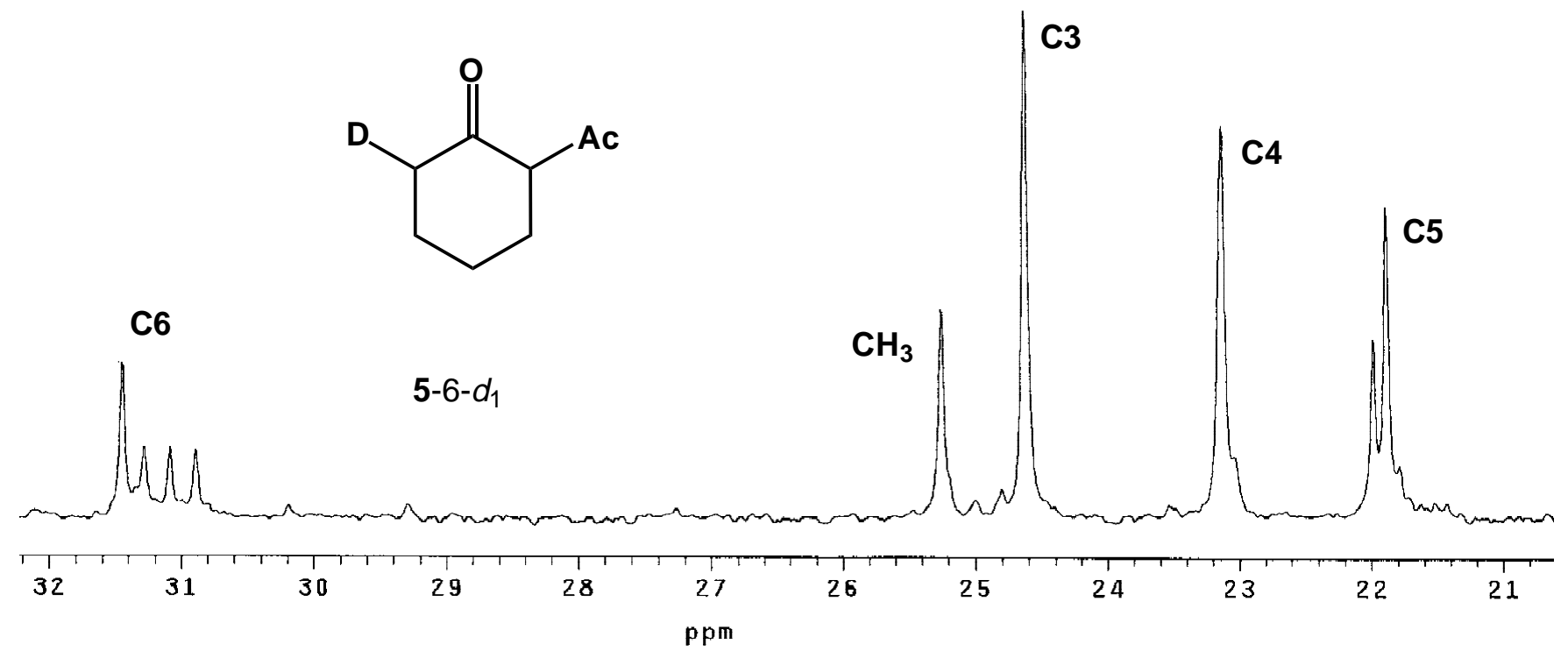

Figure S7. Aliphatic region of the ${ }^{13} \mathrm{C}\left\{{ }^{1} \mathrm{H}\right\}$ NMR spectrum of 5-2,3- $d_{2}-6-\mathrm{COCD}_{3}$.

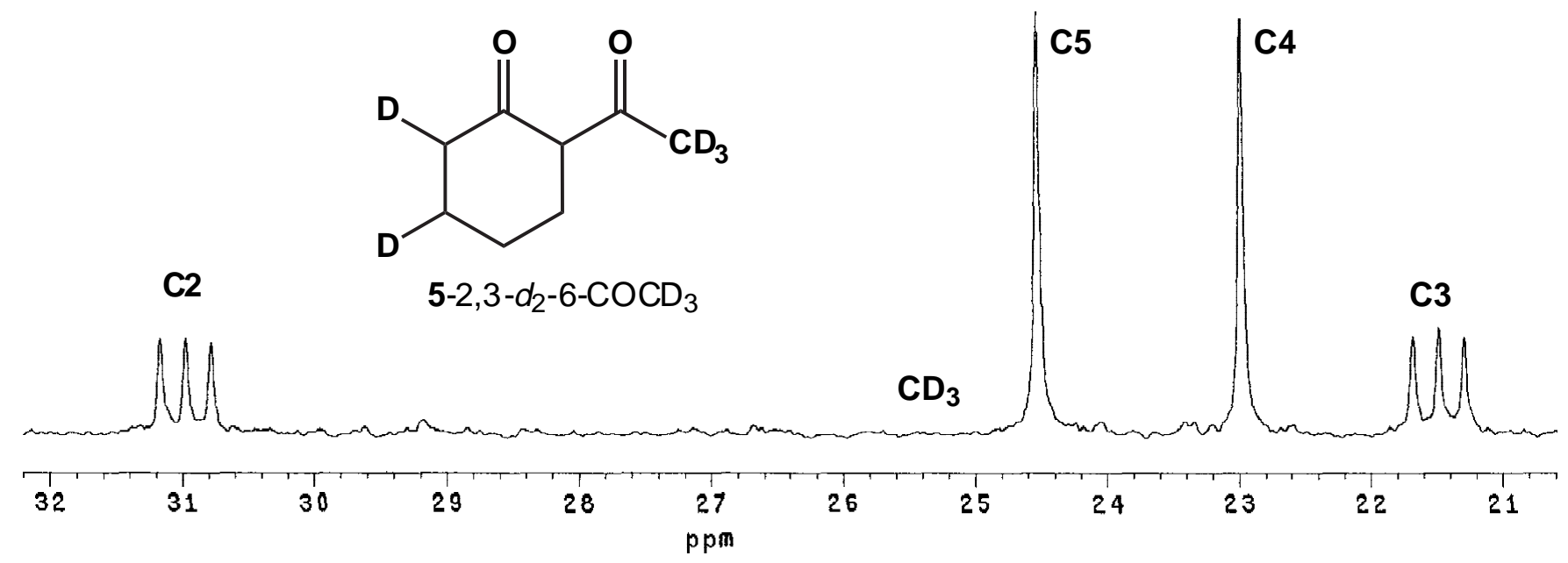


Figure S8. Aliphatic region of the ${ }^{13} \mathrm{C}\left\{{ }^{1} \mathrm{H}\right\}$ NMR spectrum of a 1:1 mixture of 5-5- $d_{1}$ and 5-4- $d_{1}$.

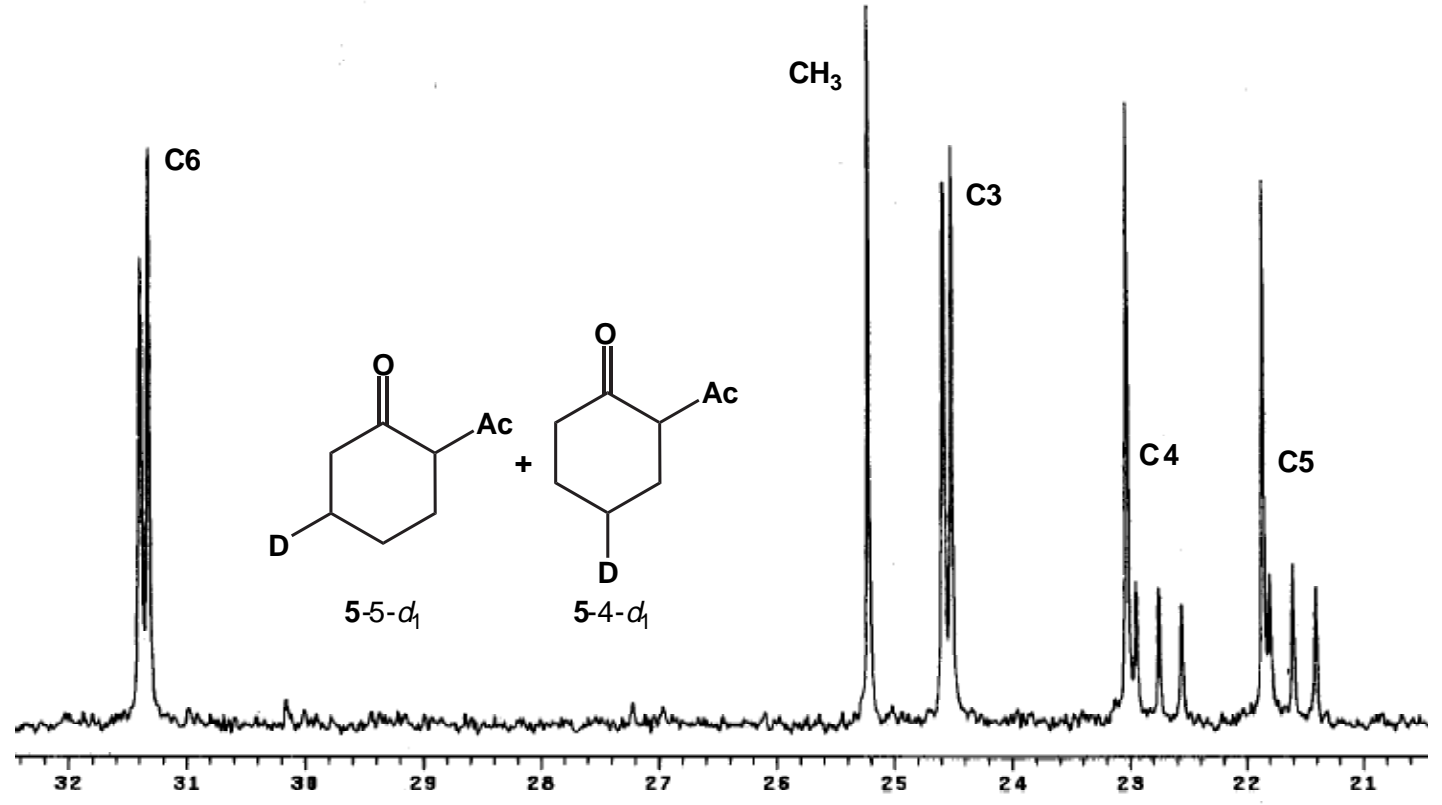

Figure S9. Aliphatic region of the ${ }^{13} \mathrm{C}\left\{{ }^{1} \mathrm{H}\right\}$ NMR spectrum of trans-5-4,5- $d_{2}$.

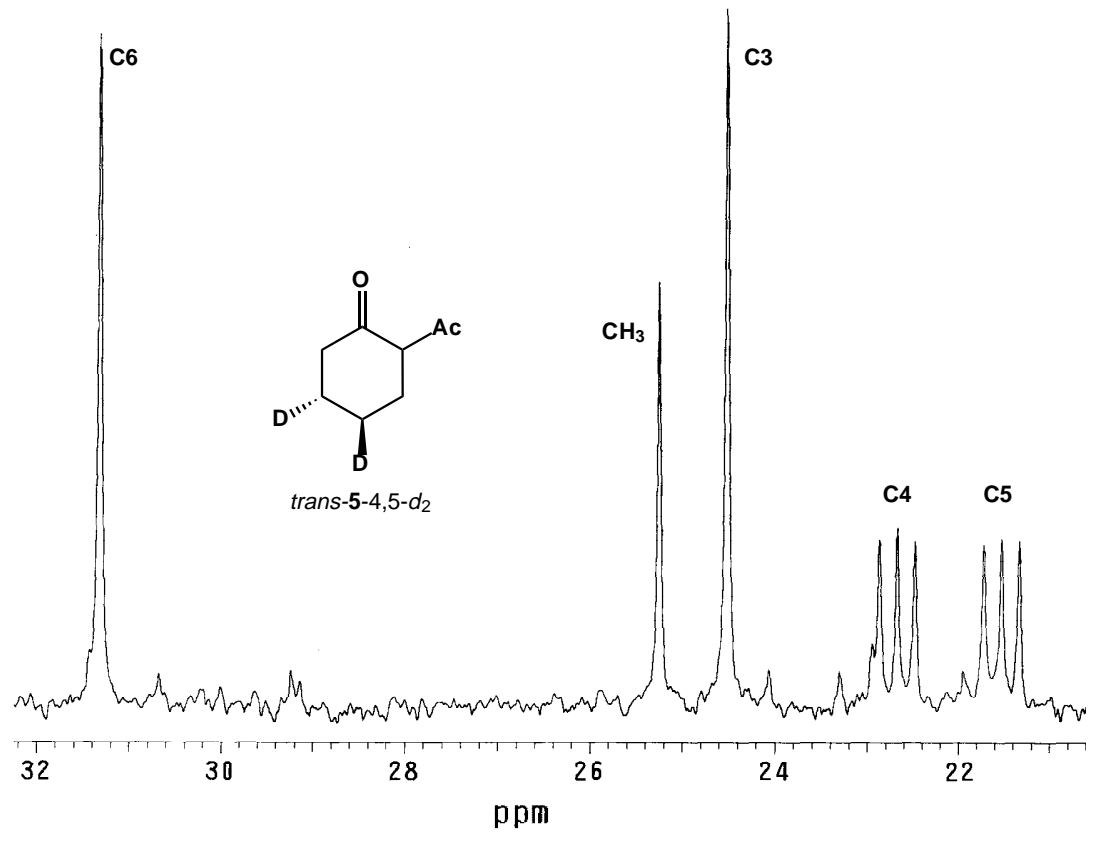


Figure S10. Aliphatic region of the ${ }^{13} C\left\{{ }^{1} \mathrm{H}\right\}$ NMR spectrum of 5-3,3- $d_{2}$.

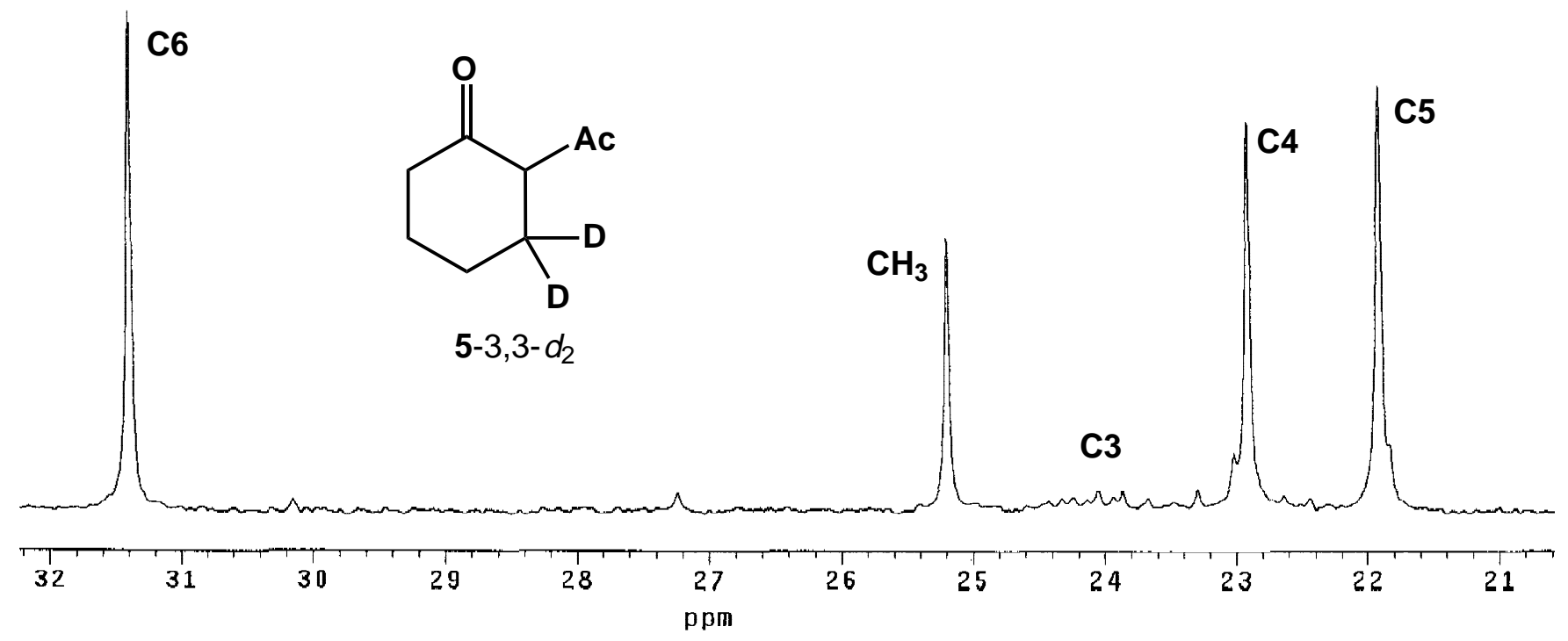

Figure S11. Aliphatic region of the ${ }^{1} \mathrm{H}_{-}{ }^{1} \mathrm{H}$ COSY NMR spectrum of 6 .

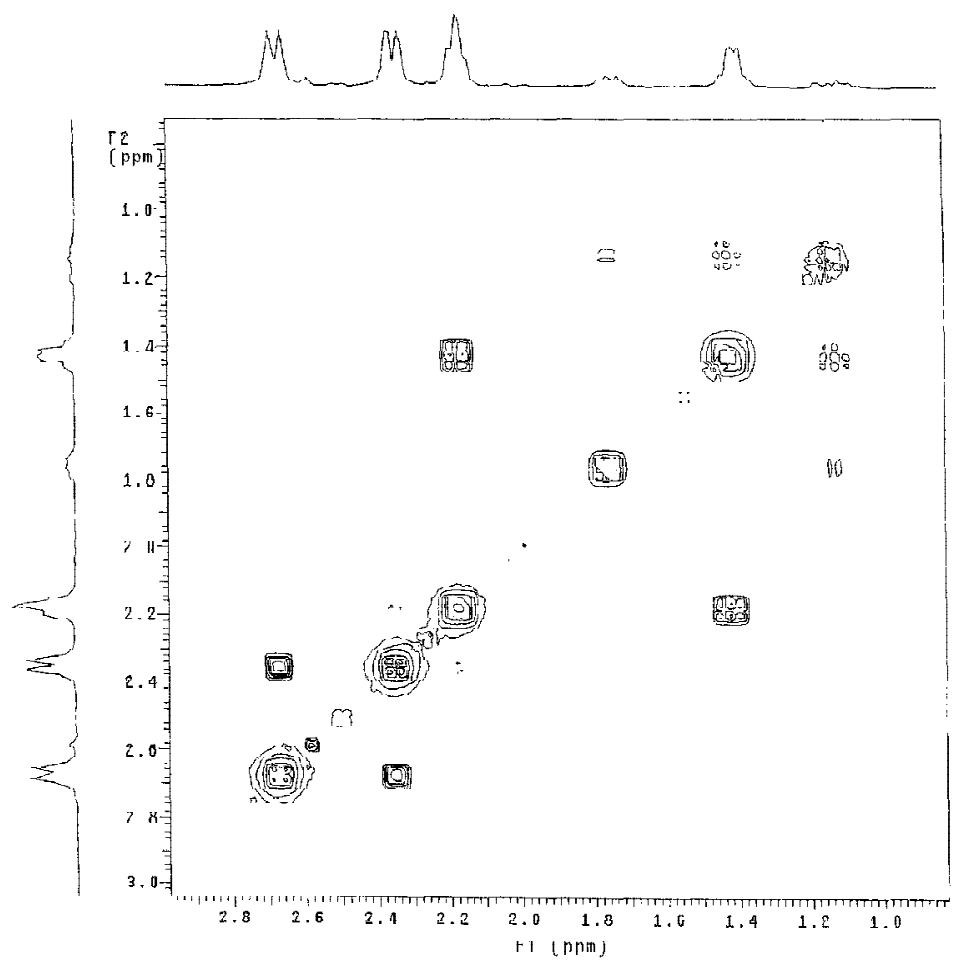


Figure S12. Aliphatic region of the ${ }^{1} \mathrm{H}$ NMR spectrum of a $~ 5: 1$ mixture of anti-6-4,5- $d_{2}$ and 6-4,5,5- $d_{3}$.

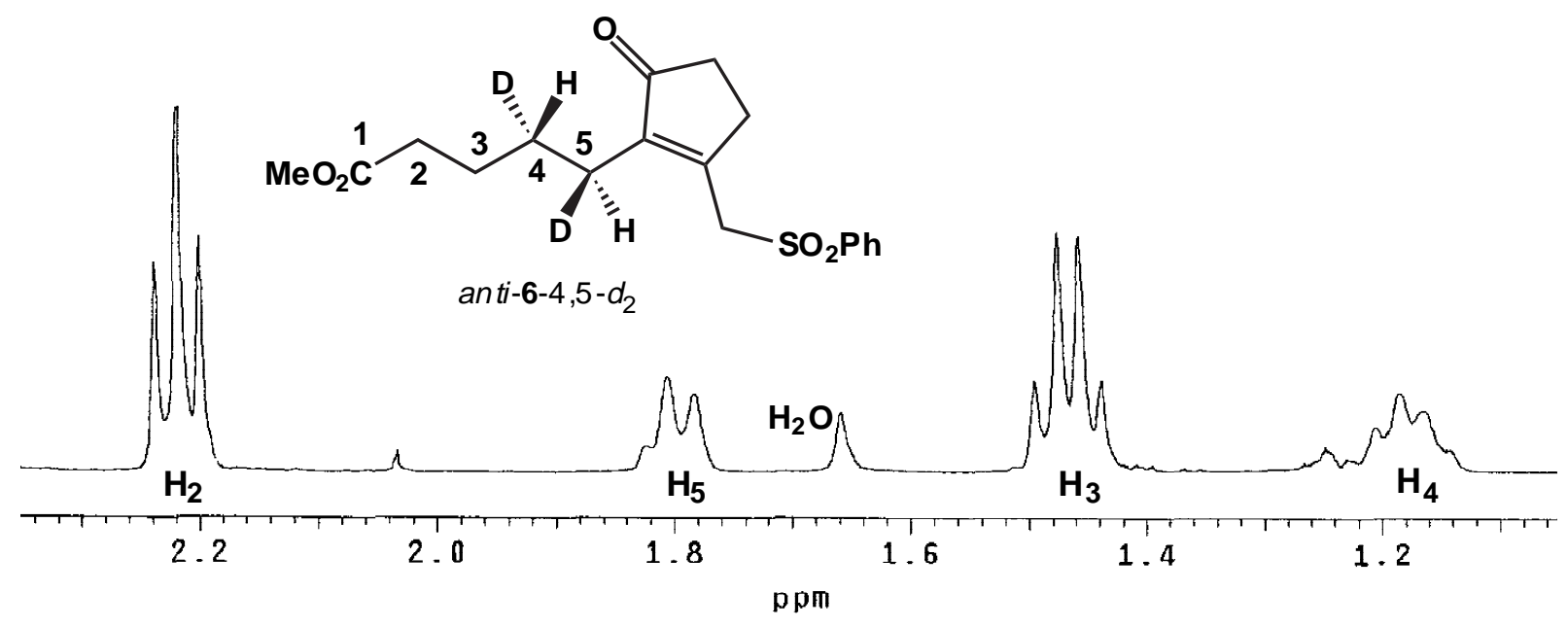

Figure S13. Aliphatic region of the ${ }^{13} \mathrm{C}\left\{{ }^{1} \mathrm{H}\right\}$ NMR spectrum of a $~ 5: 1$ mixture of anti-6-4,5-d 2 and 6$4,5,5-d_{3}$

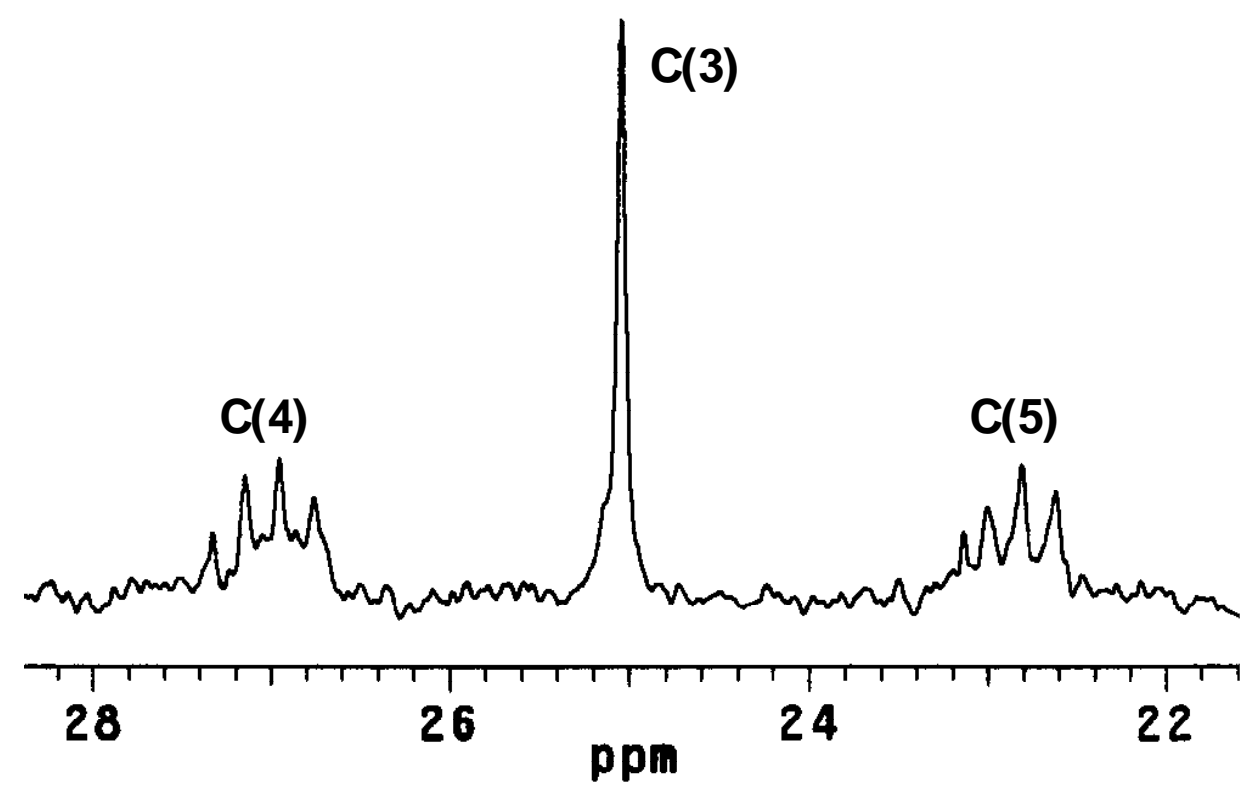


Figure S14. Aliphatic region of the ${ }^{1} \mathrm{H}$ NMR spectrum of a $~ 3: 1$ mixture of syn-6-4,5- $d_{2}$ and 6-4,5,5- $d_{3}$.

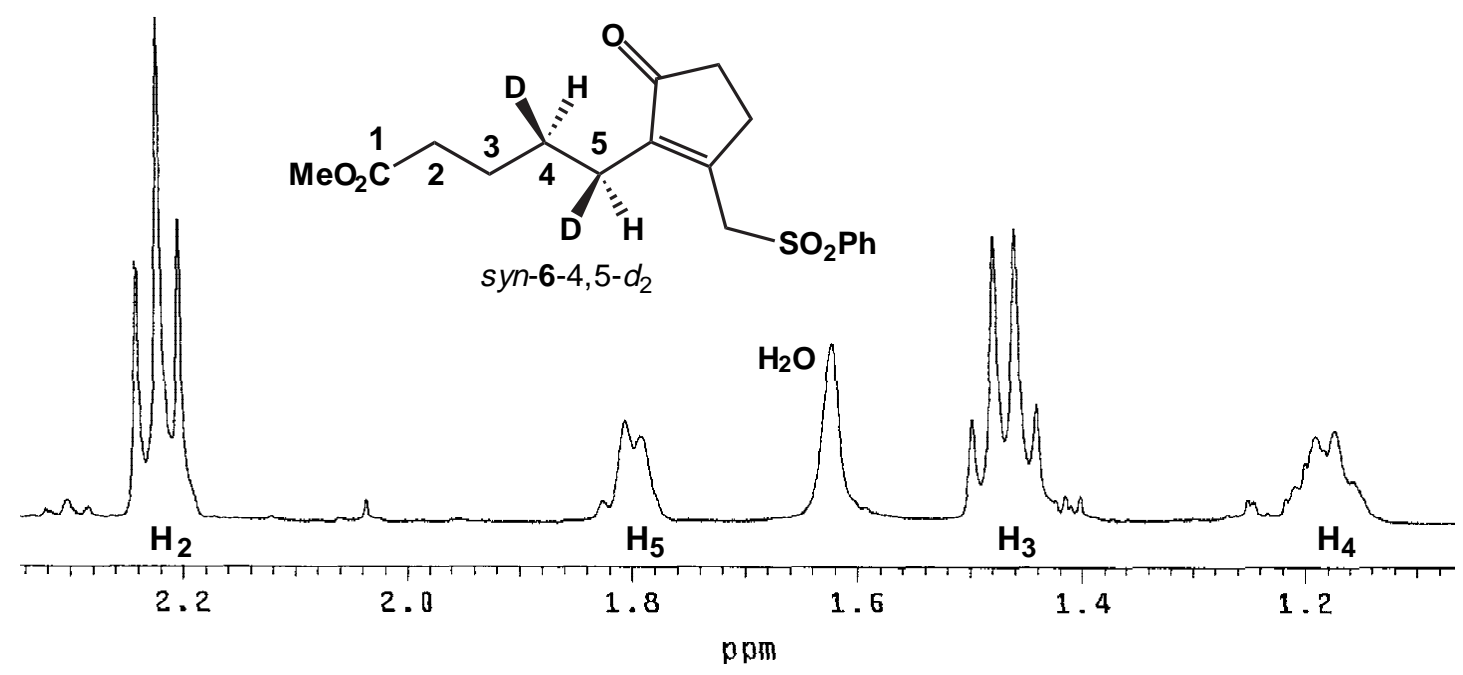

Figure S15. Aliphatic region of the ${ }^{13} \mathrm{C}\left\{{ }^{1} \mathrm{H}\right\}$ NMR spectrum of a $\sim 3: 1$ mixture of $s y n-6-4,5-d_{2}$ and 6$4,5,5-d_{3}$

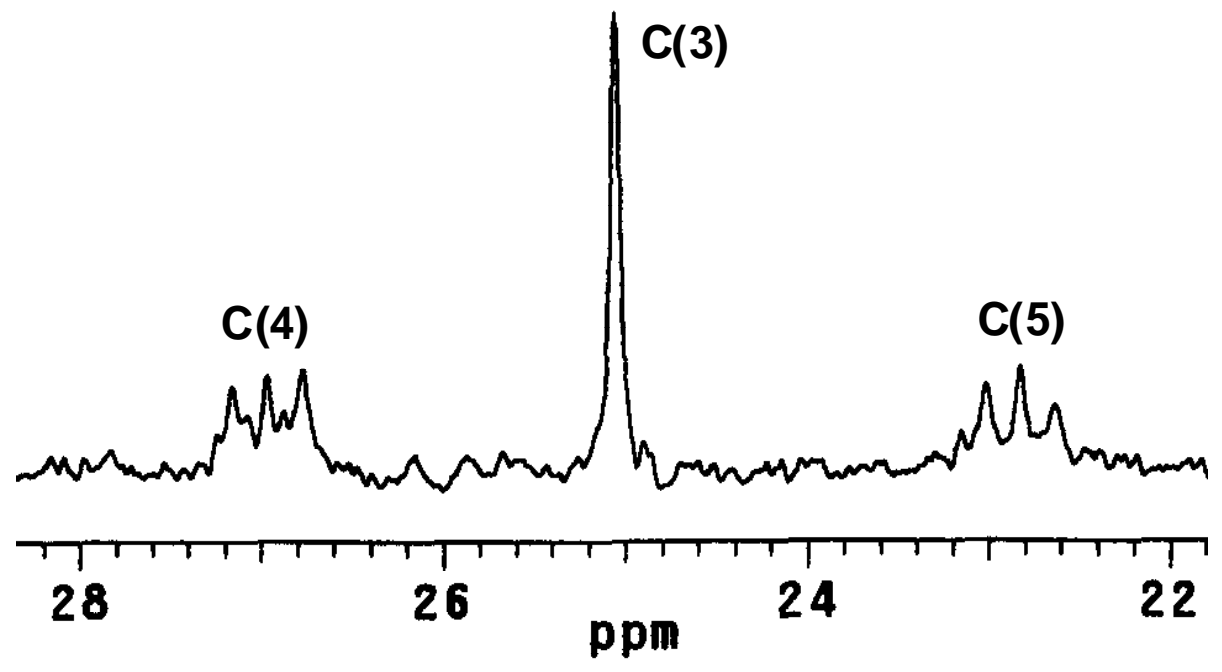


Figure S16. Aliphatic region of the ${ }^{1} \mathrm{H}$ NMR spectrum of syn-6-3,4- $d_{2}$ with selective decoupling of the $\mathrm{C}(5)$ methylene group at $\delta 1.8$.

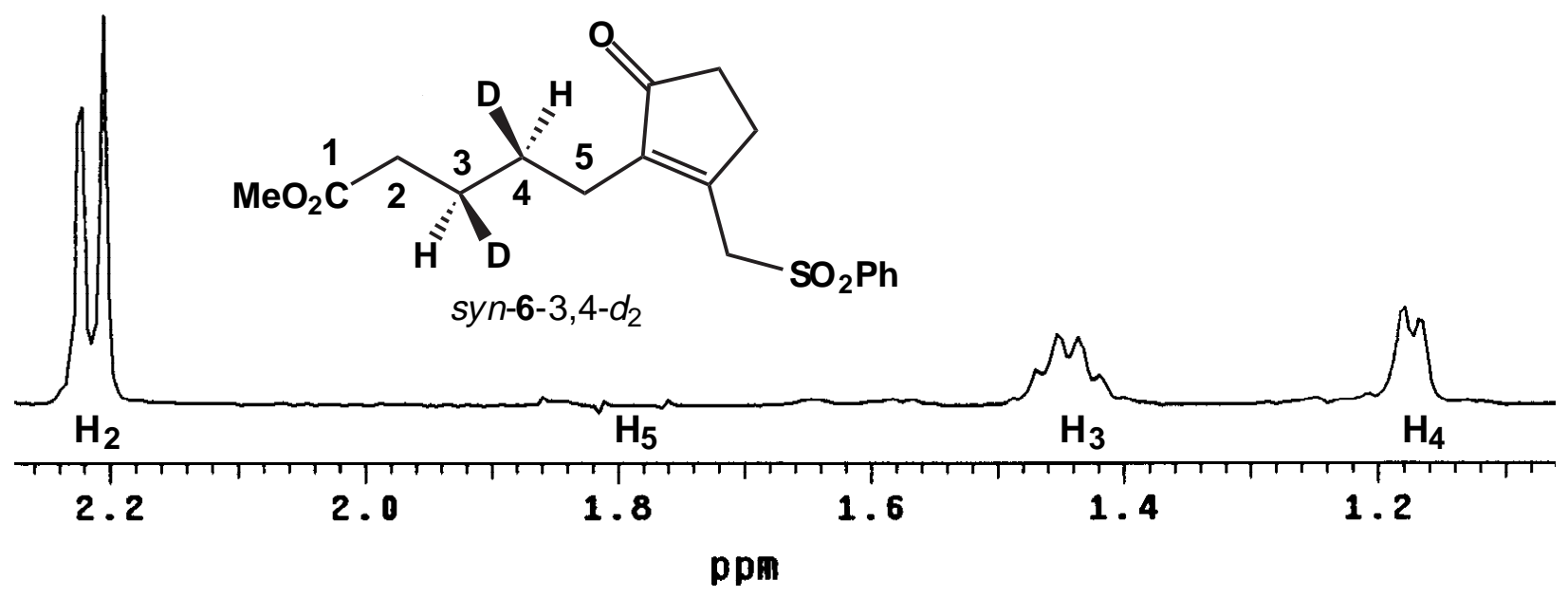

Figure S17. Aliphatic region of the ${ }^{13} \mathrm{C}\left\{{ }^{1} \mathrm{H}\right\}$ NMR spectrum of syn-6-3,4- $d_{2}$.

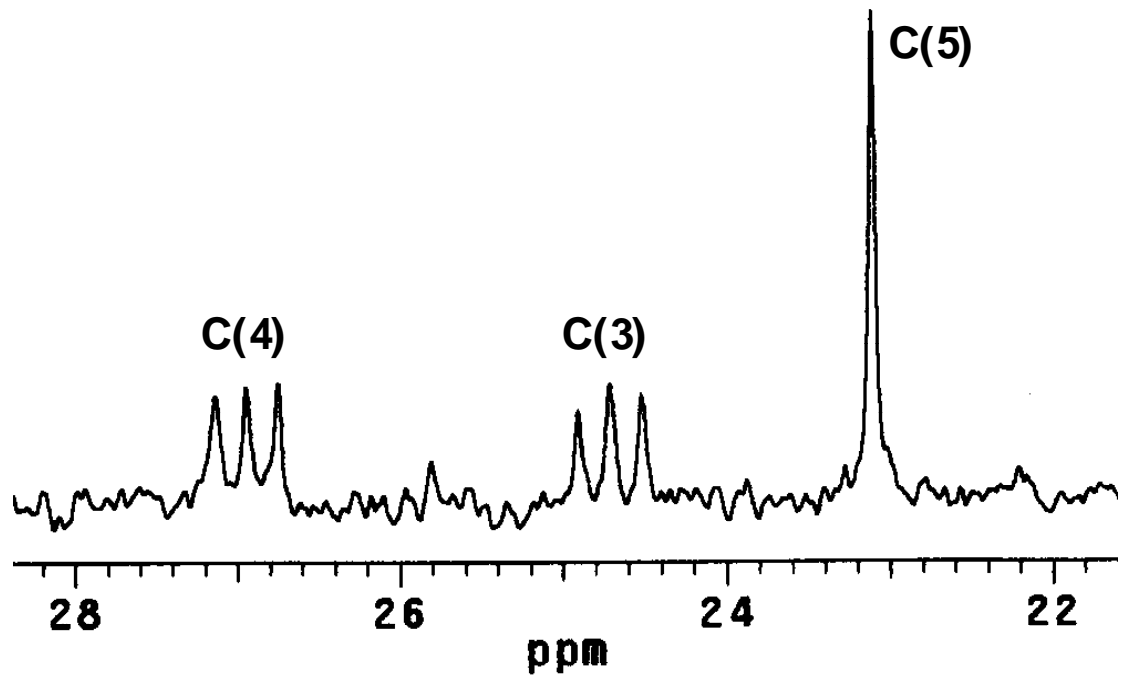




\section{References}

[1] B. B. Wayland, R. F. Schramm, Inorg. Chem. 1969, 8, 971.

[2] H. J. Reich, J. M. Renga, I. L. Reich, J. Am. Chem. Soc. 1975, 97, 5434.

[3] H. Gerlach, H. Wetter, Helv. Chim. Acta 1974, 57, 2306.

[4] E. P. Mueller, O. Jeger, Helv. Chim. Acta 1975, 58, 2173.

[5] I. Vlattas, I. T. Harrison, L. Tokes, J. H. Fried, A. D. Cross, J. Org. Chem. 1968, 33, 4176.

[6] a) L. Weiler, J. Am. Chem. Soc. 1970, 92, 6702; b) S. N. Huckin, L. Weiler, J. Am. Chem. Soc. 1974, 96, 1082.

[7] A. M. Doherty, S. V. Ley, B. Lygo, D. J. Williams, J. Chem. Soc. Perkin Trans. 1 1984, 1371.

[8] a) H. Nozaki, T. Yamaguti, T. Okada, R. Noyori, M. Kawanisi, Tetrahedron 1967, 23, 3993; b) E. C.

Taylor, G. H. Hawks, A. McKillop, J. Am. Chem. Soc. 1968, 90, 2421.

[9] A. Padwa, D. J. Austin, M. Ishida, C. L. Muller, S. S. Murphree, P. E. Yeske, J. Org. Chem. 1992, 57, 1161.

[10] K. D. McMichael, J. Am. Chem. Soc. 1967, 89, 2943.

[11] P. N. Culshaw, J. C. Walton, J. Chem. Soc., Perkin Trans. 2 1991, 1201.

[12] B. M. Trost, R. J. Kulawiec, J. Am. Chem. Soc. 1992, 114, 5579.

[13] S. Dua, M. S. Taylor, M. A. Buntine, J. H. Bowie, J. Chem. Soc., Perkin Trans 2 1997, 1991.

[14] J. E. Baldwin, M. Bradley, N. J. Turner, R. M. Adlington, A. R. Pitt, H. Sheridan, Tetrahedron 1991, 47, 8203 .

[15] R. B. Meyer, C. R. Houser, J. Org. Chem. 1960, 25, 158.

[16] J. Christoffers, Syn. Comm. 1999, 29, 117.

[17] A. B. Smith III, P. A. Levenberg, Synthesis 1981, 567.

[18] W. Oppolzer, S. Siles, R. L. Snowden, B. H. Bakker, M. Petrzilka, Tetrahedron 1985, 3497.

[19] M. Bertrand, C. Rouvier, Bull. Soc. Chim. Fr. 1968, 7, 2926.

[20] A. Balog, D. P. Curran, J. Org. Chem. 1995, 60, 337.

[21] W. Egan, G. Gunnarsson, T. E. Bull, S. Forsén, J. Am. Chem. Soc. 1977, 99, 4568.

[22] J. B. Paine, J. R. Brough, K. K. Buller, E. E. Erikson, J. Org. Chem. 1987, 52, 3986. 
[23] D. Enders, V. N. Pathak, P. Weuster, Chem. Ber. 1992, 125, 515.

[24] D. P. Curran, H. Yu, Synthesis 1992, 123.

[25] S. Bernascont, P. Gariboldt, G. Jommi, M. Sisti, Tetrahedron Lett. 1980, 21, 2337.

[26] T. Tanaka, S. Kurozumi, T. Toru, M. Kobayashi, S. Miura, S. Ishimoto, Tetrahedron 1977, 33, 1105.

[27] G. Descotes, Y. Querou, Bull. Soc. Chim. Fr. 1968, 3395.

[28] P. Stanetty, G. Purstinger, Monatsh. Chem. 1999, 130, 441.

[29] A. Padwa, Y. S. Kulkarni, Z-J. Zhang, J. Org. Chem. 1990, 55, 4144.

[30] H. Plieninger, C. E. Castro, Chem. Ber. 1954, 87, 1760. 